\title{
Super-Resolution Imaging Reveals That AMPA Receptors Inside Synapses Are Dynamically Organized in Nanodomains Regulated by PSD95
}

\author{
Deepak Nair, ${ }^{1,2 *}$ Eric Hosy, ${ }^{1,2 *}$ Jennifer D. Petersen, ${ }^{1,2}$ Audrey Constals, ${ }^{1,2}$ Gregory Giannone, ${ }^{1,2}$ Daniel Choquet, ${ }^{1,2}$ \\ and Jean-Baptiste Sibarita ${ }^{1,2}$ \\ ${ }^{1}$ University of Bordeaux, Interdisciplinary Institute for Neuroscience, and ${ }^{2}$ Centre National de la Recherche Scientifique, Unité Mixte de Recherche 5297, \\ F-33000 Bordeaux, France
}

The spatiotemporal organization of neurotransmitter receptors in postsynaptic membranes is a fundamental determinant of synaptic transmission and information processing by the brain. Using four independent super-resolution light imaging methods and EM of genetically tagged and endogenous receptors, we show that, in rat hippocampal neurons, AMPARs are often highly concentrated inside synapses into a few clusters of $\sim 70 \mathrm{~nm}$ that contain $\sim 20$ receptors. AMPARs are stabilized reversibly in these nanodomains and diffuse freely outside them. Nanodomains are dynamic in their shape and position within synapses and can form or disappear within minutes, although they are mostly stable for up to $1 \mathrm{~h}$. AMPAR nanodomains are often, but not systematically, colocalized with clusters of the scaffold protein PSD95, which are generally of larger size than AMPAR nanoclusters. PSD95 expression level regulates AMPAR nanodomain size and compactness in parallel to miniature EPSC amplitude. Monte Carlo simulations further indicate the impact of AMPAR concentration in clusters on the efficacy of synaptic transmission. The observation that AMPARs are highly concentrated in nanodomains, instead of diffusively distributed in the PSD as generally thought, has important consequences on our understanding of excitatory neurotransmission. Furthermore, our results indicate that glutamatergic synaptic transmission is controlled by the nanometer-scale regulation of the size of these highly concentrated nanodomains.

\section{Introduction}

The molecular composition and organization of synapses is a fundamental determinant of synaptic transmission. Considerable efforts have been devoted to determine precisely the spatial organization of presynaptic and postsynaptic molecular elements and to correlate it with synaptic activity. Presynaptic vesicles contain a limited number of glutamate molecules, whose release creates a transient local glutamate gradient (Lisman et al., 2007). As

\footnotetext{
Received May 17, 2012; revised June 27, 2013; accepted July 5, 2013.

Author contributions: D.N., E.H., D.C., and J.-B.S. designed research;D.N., E.H., J.D.P., A.C., and J.-B.S. performed research; G.G. and J.-B.S. contributed unpublished reagents/analytic tools; D.N., E.H., D.C., and J.-B.S. analyzed data; D.N., E.H., J.D.P., D.C., and J.-B.S. wrote the paper.

This work was supported by the Centre National de la Recherche Scientifique, the Agence Nationale pour la Recherche (StimTrafPark), the Regional Council of Aquitaine, the Fondation pour la Recherche Médicale, the European Union's seventh framework program for research and development for the Marie-Curie-Intra European Fellowship, the ERC GrantNano-Dyn-Syn, and the ERA-NET project MODIFSYN. We thank C. Breillat, D. Bouchet, B. Tessier, A. Frouin, L. Royer, and N. Retailleau for help in molecular biology and cell cultures; A. Penn for providing custom-made mEPSC analysis software; M. Heilemann for helpful advice on dSTORM; members of the Bordeaux Imaging Center, especially P. Legros for the use of the STED microscope; S. Lacomme and E. Gontier of the Electron Microscopy Facility; S. Marais for help with EM reconstructions; L. Cognet and N. Rebola for helpful discussions; and E. Gouaux for the generous gift of the anti-GluA2 antibody.

The authors declare no competing financial interests.

${ }^{*}$ D.N. and E.H. contributed equally to this work.

Correspondence should be addressed to either Dr. Daniel Choquet or Dr. Jean-Baptiste Sibarita, University of Bordeaux, Interdisciplinary Institute for Neuroscience, and Centre National de la Recherche Scientifique, Unité Mixte de Recherche 5297, 146 rue Léo Saignat, 33077, Bordeaux Cedex, France. E-mail: dchoquet@u-bordeaux2.fr or jean-baptiste.sibarita@u-bordeaux2.fr.

DOI:10.1523/JNEUROSCI.2381-12.2013

Copyright $\odot 2013$ the authors $\quad 0270-6474 / 13 / 3313204-21 \$ 15.00 / 0$
}

AMPA receptors (AMPARs) have low affinity for glutamate, only a fraction of synaptic AMPARs are activated by this gradient (Liu et al., 1999). Thus, the precise localization of AMPARs with respect to the presynaptic terminal and glutamate release sites is crucial to tune synaptic transmission. Modeling and experimental data indicate that heterogeneity of AMPAR distribution may have a strong impact on synaptic transmission (Glavinovic, 1999; Franks et al., 2002; Franks et al., 2003; Xu-Friedman and Regehr, 2004; Wu et al., 2007; Tarusawa et al., 2009; Budisantoso et al., 2012). Three categories of approaches have been used to determine AMPAR organization: light or electron microscopy (EM) and electrophysiology. All have concluded that AMPARs are concentrated in the postsynaptic density (PSD), directly opposed to presynaptic terminals (Baude et al., 1995; Kharazia and Weinberg, 1997; Takumi et al., 1999b). AMPARs are also present in the extrasynaptic membrane and in intracellular pools, and constant exchange of AMPARs between these different pools by lateral diffusion and recycling tunes receptor numbers at synapses and regulates synaptic transmission (Luscher et al., 1999; Makino and Malinow, 2009; Petrini et al., 2009; Choquet, 2010).

The precise distribution of AMPARs in nanometer-sized subdomains has so far only been studied in fixed samples. Immunogold labeling of AMPARs for EM is the highest-resolution imaging technique (Kharazia et al., 1996; Petralia et al., 1997; Nusser et al., 1998; Takumi et al., 1999a). However, the exact distribution of AMPAR density is still controversial. Studies demonstrate either that synaptic AMPARs are localized at the 
PSD periphery (Kharazia et al., 1996; Matsubara et al., 1996; Bernard et al., 1997; Kharazia and Weinberg, 1997; Chen et al., 2008) or randomly distributed (Nusser et al., 1994; Masugi-Tokita and Shigemoto, 2007; Masugi-Tokita et al., 2007). Pre-embedding immunogold EM and replica-based labeling indicate the existence of AMPAR clusters on the membrane (Masugi-Tokita et al., 2007; Tarusawa et al., 2009; Tao-Cheng et al., 2011; Budisantoso et al., 2012). Complementary to EM, super-resolution light microscopy of immunofluorescently labeled samples, which allows breaking the diffraction barrier to $\sim 250 \mathrm{~nm}$, has revealed the molecular organization of postsynaptic molecules at nanometric resolution (Dani et al., 2010). In many synapses, AMPARs were organized centrally, whereas in others they were organized laterally to the PSD. We recently performed mathematical analysis of live super-resolution tracking of AMPAR movements and found that AMPARs move to attracting interaction potential wells at the subdiffraction level in hippocampal dendrites (Hoze et al., 2012).

Here, we performed a detailed analysis of AMPAR and PSD95 localization and mobility at synapses using a combination of high-resolution imaging approaches. We demonstrate that AMPARs are strongly nonuniformly organized in synapses.

\section{Materials and Methods}

Constructs. mEos2::GluA1 (noted Eos::GluA1), GluA2::tdEos (noted Eos::GluA2), and Homer1c::Cerulean were subcloned from SEP::GluA1, GluA2::GFP, Homer1C::Dsred, and Homer1c::GFP, which were described previously (Saglietti et al., 2007; Opazo et al., 2010). Eos::TEV::GluA1 was modified from mEos2::GluA1 by inserting a Tobacco Etch Virus Protease recognition site comprising a seven amino acid sequence (Glu-Asn-Leu-Tyr-Phe-Gln-Gly, cleaving between Gln and Gly), for removing Eos tag from surface-expressed Eos::GluA1 of live neurons. PSD95::mEos2 (noted Eos::PSD95) was subcloned from PSD95::EGFP (used for overexpression experiments), which was described previously (Mondin et al., 2011). The GFP-tagged shRNA construct designed to silence expression of PSD95 (SH::PSD95) and the PSD95 rescue construct (Rescue::PSD95), which expresses shRNA against PSD95 simultaneously with an shRNA-resistant form of GFPtagged PSD95, were characterized previously (Schluter et al., 2006; Mondin et al., 2011). A generic scrambled RNA (SH::Scramble) vector containing a noneffective mammalian protein coexpressed either with GFP (SH::Scramble::GFP) or Homer1c::GFP (SH::Scramble::Homer1c) was used as a negative control to compare the effect of SH::PSD95::GFP. The compensatory mutant for stagazin and PSD95 were described and characterized previously (Schnell et al., 2002; Bats et al., 2007). The Stargazin/PSD95 compensatory mutants fused with GFP were obtained by modifying the -2 position threonine of Stargazin to phenylalanine (StgT231F) and the 225 position histidine of PSD-95 to valine (PSD95H225V).

Cell culture and transfection. Preparation of cultured neurons was performed as previously described (Heine et al., 2008). Hippocampal neurons from 18-d-old rat embryos of either sex were cultured on glass coverslips following the Banker protocol (Kaech and Banker, 2006). Neurons were transfected using Effectene at 9-11 d in vitro (DIV) or by Nucleofection at the time of plating (Nucleofector II Device, Lonza Cologne) with mEos2::GluA1, Eos::TEV::GluA1, GluA2::tdEos, and PSD95::mEos2, alone or in combination with Homer1c::Cerulean, HA::GluA1, or Homer1c::GFP, and experiments were performed at 14-20 DIV.

Single particle tracking photoactivation localization microscopy (spt$P A L M$ ). Cells were imaged at $37^{\circ} \mathrm{C}$ in an open chamber (Ludin chamber, Life Imaging Services) mounted on an inverted motorized microscope (Nikon) equipped with a $100 \times 1.49$ NA PL-APO objective and a perfect focus system, allowing long acquisition in oblique illumination mode. Imaging was performed for $<20 \mathrm{~min}$ unless otherwise stated in an extracellular solution as in Petrini et al. (2009). Cells expressing Eos::GluA1 or Eos::GluA2 were photoactivated using a $405 \mathrm{~nm}$ laser (Omicron), and the resulting photoconverted single molecule fluorescence was excited with a
$561 \mathrm{~nm}$ laser (Cobolt). Both lasers illuminated the sample simultaneously. Their respective power was adjusted to keep the number of the stochastically activated molecules constant and well separated during the acquisition. Laser intensities were tuned to leave the single molecule fluorescent during multiple frames before bleaching. The fluorescence was collected by the combination of a dichroic and emission filters (D101-R561 and F39-617, respectively, Chroma) and a sensitive EMCCD camera (Evolve, Photometric). The acquisition was steered by MetaMorph software (Molecular Devices) in streaming mode at 50 frames per second ( $20 \mathrm{~ms}$ exposure time) using a $200 \times 200$ pixels region of interest. The native nonactivated fluorescence form of Eos molecules was excited using a conventional GFP filter cube (ET470/40, T495LPXR, ET525/50, Chroma). Homer1c::Cerulean fluorescent protein was observed using a CFP filter (ET436/20, T455LP, ET480/40, Chroma). Multicolor fluorescent micro-beads (Tetraspeck, Invitrogen) were used as fiduciary markers to register long-term acquisitions and correct for lateral drifts.

Universal point accumulation in nanoscale topography (uPAINT). uPAINT was applied as reported previously (Giannone et al., 2010). Dissociated neurons were nucleofected at the time of plating with Homer1c::GFP, allowing synapse identification. Experiments took place at 13-15 DIV. Coverslips were mounted on a Ludin chamber filled with $600 \mu \mathrm{l}$ of extracellular solution at $37^{\circ} \mathrm{C}$ as in Petrini et al. (2009). A low concentration of ATTO-647 nm-coupled anti-GluA2 antibodies was then added to the chamber. Stochastic labeling of endogenous GluA2containing AMPA receptors by dye-coupled antibodies allowed us to record thousands of trajectories lasting longer than $1 \mathrm{~s}$. The amount of nonspecific binding of antibodies was evaluated using hippocampal cultures derived from GluA2 knock-out mice and was found to be $\sim 15 \%$. Potential effects of the antibody binding on receptor dynamics was tested by comparing two different GluA2 antibodies and results were found to be similar (see Fig. 6A,B). Multicolor fluorescent micro-beads (Tetraspeck, Invitrogen) were used as fiduciary markers to register long-term acquisitions and correct for lateral drifts.

Single molecule localization and tracking. A typical single-cell sptPALM or uPAINT experiment acquired with the microscope setup and protocol described above produced a set of 20,000 images that were analyzed to extract molecule localization and dynamics. Single molecule fluorescent spots were localized in each image frame and tracked over time using a combination of wavelet segmentation (Izeddin et al., 2012) and simulated annealing (Racine et al., 2006, 2007) algorithms. Under the experimental conditions described above, the image resolution of the experimental setup was quantified to $46.6 \mathrm{~nm}$ for sptPALM. The localization accuracy, which depends on the image signal-to-noise ratio and the segmentation algorithm (Kubitscheck et al., 2000; Cheezum et al., 2001; Izeddin et al., 2012), was determined experimentally using fixed samples expressing Eos-tagged proteins. We analyzed 133 2D distributions of single molecule positions belonging to long trajectories $(>50$ frames) by bidimensional Gaussian fitting, and the resolution was determined to be $2.3 \sigma$. The software package used to derive quantitative data on protein localization and dynamics is custom written as a plug-in running within the MetaMorph software environment. For the trajectory analysis, synapses were identified by wavelet image segmentation of the Homer 1c postsynaptic marker. The corresponding binary mask was then used to sort single particle data analyses to specific synaptic regions. Dendrites were identified as subcellular regions lacking the synaptic marker.

Live-cell surface staining and stimulated emission depletion microscopy (STED). The monocolonal antibody against an extracellular epitope of GluA2 was prepared as described previously (Giannone et al., 2010). Primary neuronal cultures transfected with Homerlc::GFP were incubated with the monoclonal anti-GluA2 antibody $(3 \mu \mathrm{g} / \mathrm{ml})$ in the neuronal growth medium for $4-6 \mathrm{~min}$ at $37^{\circ} \mathrm{C}$, and then fixed in $4 \%$ paraformaldehyde and $4 \%$ sucrose in PBS. Fixed samples were rinsed in PBS and then blocked in PBS containing $1 \%$ BSA. The primary antibodies were then revealed by incubating ATTO647N (ATTO-tec) coupled anti-mouse IgG secondary antibodies for $30 \mathrm{~min}$ at room temperature. Finally, samples were rinsed in PBS and mounted in Vectashield mounting medium. We used a commercial STED inverted microscope (DMI6000 TCS SP5 AOBS, Leica) to obtain 
super-resolved images of GluA2-ATTO647N-labeled neurons expressing Homer1c::GFP as a postsynaptic marker. The Homer1c::GFP images were recorded using the regular confocal mode on the same microscope. A spatial resolution of $63.7 \pm 1.2 \mathrm{~nm}$ was measured using $40 \mathrm{~nm}$ crimson beads.

Direct stochastic optical reconstruction microscopy (dSTORM). Primary neuronal cultures were incubated with rabbit-anti-GluA1 antibody (targeted to the extracellular region RTSDSRDHTRVDWKRC, Agro-Bio) for 20-30 min. They were then fixed using $4 \%$ paraformaldehyde and $4 \%$ sucrose in PBS, and washed with PBS. They were incubated with $\mathrm{NH}_{4} \mathrm{Cl} 50 \mathrm{~mm}$ for $30 \mathrm{~min}$ before permeabilization. They were permeabilized using $0.1 \%$ Triton X-100 and incubated with PBS containing 1\% BSA for $30 \mathrm{~min}$. They were then incubated with mouse-anti-PSD95 antibody (MA1-046, Thermo Fisher Scientific) for $30 \mathrm{~min}$ and washed several times with PBS containing $1 \%$ BSA. The primary antibodies were then revealed by incubating Alexa-647-coupled anti-rabbit IgG secondary (A21245, Invitrogen) and RhodamineRed coupled anti-mouse secondary antibodies (715-295-151, Jackson ImmunoResearch Laboratories) for 30 $\mathrm{min}$ at room temperature. For single-color dSTORM experiments, primary neuronal cultures were incubated with mouse-anti-GluA2 antibody (Giannone et al., 2010) for 5-7 min. They were then fixed, as described previously. The primary antibodies were then revealed by incubating Alexa-647 coupled anti-mouse IgG secondary (Invitrogen) for $30 \mathrm{~min}$ at room temperature.

The stained coverslips were imaged the next day at room temperature in a closed chamber (Ludin Chamber, Life Imaging Services) mounted on an inverted motorized microscope equipped with a $100 \times 1.49 \mathrm{NA}$ PL-APO objective and a Perfect Focus System (Nikon), allowing long acquisition in oblique illumination mode. Imaging was performed in an extracellular solution containing reducing agents and oxygen scavengers. For dSTORM, ensemble fluorescence of Rhodamine Red and Alexa-647 was first converted in to dark state using a $561 \mathrm{~nm}$ laser or $640 \mathrm{~nm}$ laser (Coherent) at $30-50 \mathrm{kw} / \mathrm{cm}^{2}$ intensity. Once the ensemble fluorescence was converted into the desired density of single molecules per frame, the laser power was reduced to $7-15 \mathrm{kw} / \mathrm{cm}^{2}$ and imaged continuously at 50 fps for 20,000 frames. The level of single molecules per frame was controlled by using a $405 \mathrm{~nm}$ laser (Omicron). The dyes were sequentially imaged (Alexa-647 first and followed by Rhodamine) to collect the desired single molecule frames. The laser powers were adjusted to keep an optimal level of stochastically activated molecules during the acquisition. Both the ensemble and single molecule fluorescence was collected by the combination of a dichroic and emission filter (D101-R561 and F39-617, respectively, Chroma; and quad-band dichroic filter (Di01-R405/488/ $561 / 635$, Semrock). The fluorescence was collected using a sensitive EMCCD (Evolve, Photometric). Single molecule localization and reconstruction were performed online using automatic feedback control on the lasers, enabling optimal molecule density during the acquisition (Kechkar et al., 2013). The acquisition and localization sequences were driven by MetaMorph software (Molecular Devices) in streaming mode at 50 frames per second ( $20 \mathrm{~ms}$ exposure time) using an area equal to or $<256 \times 256$ pixels region of interest. We used multicolor fluorescent microbeads (Tetraspeck, Invitrogen) as fiduciary markers to register long-term acquisitions and correct for lateral drifts and chromatic shifts. A spatial resolution of $14 \mathrm{~nm}$ was measured using centroid determination on $100 \mathrm{~nm}$ Tetraspeck beads acquired with similar signal-to-noise ratio than dSTORM single molecule images.

Synaptic surface area quantification. Epifluorescence images of the synaptic marker protein Homer1c (Homer1c::GFP or Homer1c::Cerulean) were used to differentiate between synapses and the rest of the dendrite. Epifluorescence images of Homerlc were first thresholded and segmented using the morphometric image analysis module of MetaMorph software (Molecular Devices) for structures $>0.02 \mu \mathrm{m}^{2}$. Morphological features, such as area, length, and breadth of each segmented structure, were exported to calculate their respective distributions. The mean synaptic area of Homer 1c labeled structures was measured to be $0.11 \pm 0.04$ $\mu \mathrm{m}^{2}$, which is consistent with the surface area of the spine head. The Homer1c segmented images were then used to define synapses area for single particle tracking experiments.

Super-resolution cluster analysis. AMPAR nanodomains and PSD95 subclusters were identified from super-resolution images by custom software written as a plug-in running inside MetaMorph. Single-molecule- based super-resolution images were reconstructed from the 20,000 frames before being analyzed, whereas STED bulk images were analyzed raw. Nanodomains, which corresponded to clustered areas where the signal density was higher, were first identified by wavelet segmentation. Nanodomain number and dimensions were then computed by $2 \mathrm{D}$ anisotropic Gaussian fitting, from which the principal and the auxiliary axes were extracted as $2.3 \sigma_{\text {long }}$ and $2.3 \sigma_{\text {short }}$, respectively. The shape factor was calculated as a ratio between the auxiliary and the principal axes.

For the quantification of PSD95 cluster and nanodomain surface areas, the single molecule-based super-resolution images were first segmented by the morphometric image analysis module of MetaMorph software for structures $>0.02 \mu \mathrm{m}^{2}$. Morphologic features, such as area, length, and breadth of each cluster, were exported to calculate their respective distribution. The dimensions of PSD95 subclusters were calculated the same way as AMPAR nanodomains.

Proteolytic cleavage assay of extracellular Eos. The proportion Eos::TEV::GluA1 that was localized on neuronal surface was assessed using AcTEV Protease (Invitrogen) to cleave the Eos tag from surface expressed receptors. sptPALM was used to measure fluorescence intensity of Eos::TEV::GluA1-expressing cells before and after incubation with AcTEV protease in extracellular medium (1:60 dilution in presence of 1 $\mathrm{mM}$ DTT, for $4-5 \mathrm{~min}, 37^{\circ} \mathrm{C}$ ). Control neurons were incubated in AcTEV protease, which was rendered inactive by boiling for $10 \mathrm{~min}$.

Live cell nanogold immunolabeling and electron microscopy of cultured neurons. Coverslips with attached neurons (DIV 18-21) were incubated for $3 \mathrm{~min}$ at $37^{\circ} \mathrm{C}$ in GluA2 monoclonal antibody diluted in culture medium $(6 \mu \mathrm{g} / \mathrm{ml}$, gift from E. Gouaux). Neurons were removed from antibody and fixed in freshly prepared, prewarmed EM-grade $4 \%$ paraformaldehyde (EMS) in 0.15 M Sorensen's phosphate buffer (EMS) at room temperature for $45 \mathrm{~min}$. All subsequent steps were performed at room temperature. Neurons were rinsed 3 times in $0.15 \mathrm{M}$ Sorensen's PB, once in $0.1 \mathrm{M}$ Millonig's PBS, and then blocked in $0.1 \mathrm{M}$ Millonig's PBS with $2 \%$ BSA and $0.1 \%$ cold water fish skin gelatin (Aurion, EMS) for 60 min. Next, neurons were incubated in FluoroNanogold anti mouse Fab' AlexaFluor-488 (Nanoprobes) diluted 1:100 in 0.1 M Millonig's PBS blocking solution for $90 \mathrm{~min}$, then rinsed once in blocking solution, once in Sorensen's PB, and placed in freshly prepared $2 \%$ glutaraldehyde (EMS) in Sorensen's PB for 30 min. Afterward, neurons were stored in Sorensen's PB until silver intensification.

Processing and embedding for electron microscopy. FluoroNanogold label was enhanced for 6-8 min using HQ Silver Reagent (Nanoprobes) according to manufacturer's instructions and processed immediately for $\mathrm{EM}$; all steps were performed at room temperature. After several rinses in Sorensen's $\mathrm{PB}$, neurons were incubated in $0.2 \% \mathrm{OsO}_{4}$ in Sorensen's $\mathrm{PB}$ for $30 \mathrm{~min}$, and then rinsed 10 times in $\mathrm{dH}_{2} \mathrm{O}$ to remove all traces of $\mathrm{PB}$ before placing neurons in filtered $0.25 \%$ uranyl acetate dissolved in $\mathrm{dH}_{2} \mathrm{O}$ for $30 \mathrm{~min}$. After several water rinses, neurons were dehydrated by 3 min incubations in a graded series of ethanol: 50\%, 70\%, 95\%, and $100 \%$. No propylene oxide was used to prevent loss of immunogold label. Samples were infiltrated during 1-2 h steps in 70\% Epon812/ethanol mixture followed by 2 exchanges of $100 \%$ freshly prepared Epon 812 (Taab Laboratory and Microscopy), and finally embedded in freshly prepared Epon812. To allow cutting of en face sections of neurons, coverslips were placed cell side facing up on a glass slide and gelatin capsules filled with Epon 812 were inverted and placed on top of coverslip, and polymerized at $60^{\circ} \mathrm{C}$ for $48 \mathrm{~h}$. Coverslips were removed from polymerized samples by gentle heating over a flame while pulling slightly on the glass slide and gelatin capsules. Ultrathin sections $(60-70 \mathrm{~nm})$ were cut using an Ultra $35^{\circ}$ diamond knife (Diatome) and a Leica Ultracut UCT M26 (Leica Microsystems) and picked up on $2 \mathrm{~mm}$ slot grids with a $1 \%$ formvar support film (EMS). When cutting serial sections, a 50:50 mix of rubber cement/xylene was applied to the top and bottom sides of the trimmed block to prevent sections from separating. Sections were contrasted with $3 \%$ aqueous uranyl acetate for $5 \mathrm{~min}$, and then Reynolds's lead citrate for $7 \mathrm{~min}$ before imaging using a Hitachi H7650 transmission electron microscope operated at $80 \mathrm{kV}$. Images were captured using an Orius CCD (Gatan).

Analysis of the pattern and distribution of immunogold labeling on $2 D$ EM sections. Micrographs were analyzed using MetaMorph software 
(Molecular Devices). To quantify the density of immunolabel on different membrane domains, ROIs were drawn along membranes that were categorized as follows: spine PSD membrane, extrasynaptic spine membrane, or dendritic shaft membrane. For each ROI, the surface was measured and the number of immunolabels that were within $20 \mathrm{~nm}$ of the membrane was counted. The size of the clusters was measured using the Integrated Morphometry Analysis module, after adjusting the threshold to select label from the background. Immunolabel particles that were overlapping or separated by $<20 \mathrm{~nm}$ were identified as a cluster.

$3 D$-reconstruction of synaptic spines by serial section EM. Series of images taken at 40,000-50,000 magnification from 6 to 8 serial sections of $70 \mathrm{~nm}$ thickness were used to generate reconstructions of individual immunogold labeled spines. Stacks of images were aligned using the MetaMorph software auto-align module. Reconstructions were generated from the aligned stacks using Imaris software (Bitplane). The membrane contours of the spine were traced in each section to create the spine volume. Immunolabel was reconstructed using the Spots tool. The number and size of nanodomains per spine were measured on each image of the serial sections as previously described. Label that appeared in the same location in successive serial sections was considered to be the same immunolabel and counted only once.

Quantification of AMPAR content in nanodomains. The number of AMPARs per nanodomain was estimated from dSTORM superresolution reconstructions of endogenous GluA2 using a custom-made analysis module operating inside MetaMorph software. For each cell, single AMPARs were identified using wavelet segmentation and Gaussian fitting as isotropic and isolated objects of size $<40 \mathrm{~nm}$ in diameter. Isolated single AMPARs could be differentiated from nanoclusters resulting from the high spatial resolution provided by the dSTORM imaging technique. Then, the histogram and median of the integrated intensity of each individual AMPAR per cell were computed. This corresponds to the intensity distribution of one tetrameric structure, in function of the ratio of immunolabeled GluA2 with the other nonlabeled AMPAR subunits. Finally, synaptic nanodomains were detected using wavelet segmentation similarly to the cluster analysis described in Materials and Methods, and the number of receptors per cluster was estimated by dividing the cluster's total intensity by the median intensity of the identified isolated AMPARs.

Electrophysiology. The extracellular recording solution contained in mu concentration as follows: $130 \mathrm{NaCl}, 2.5 \mathrm{KCl}, 2 \mathrm{MgCl}_{2}, 2 \mathrm{CaCl}_{2}, 10$ HEPES, and 10 D-glucose, $\mathrm{pH}$ 7.4. To block GABA-A receptors, $50 \mu \mathrm{M}$ picrotoxin was added to solution, and mini-EPSC recordings were performed in the presence of $1 \mu \mathrm{M}$ TTX. The bath temperature was kept at $34^{\circ} \mathrm{C}$. Patch-clamp microelectrodes were pulled for a $4-6 \mathrm{M} \Omega$ resistance, from borosilicate on a P-97 model puller (Sutter Instruments). Mini recordings were performed with an EPC 10 double patch-clamp amplifier (HEKA Elektronik). Data analysis was performed with a homemade software (Penn et al., 2012). Cells are clamped at $-70 \mathrm{mV}$.

Statistics. Statistical values are given as medians $\pm 25 \% / 75 \%$ interval or mean \pm SEM, except for Figure 10 where mean \pm SD is used. Statistical significances were performed using Sigmaplot software (Systat Software). Non-Gaussian distribution datasets were tested by MannWhitney $U$ test, and Gaussian distributions were tested by $t$ test.

Calculation of required sample size. The experiments were designed to compare two conditions per sample, including a control condition. The experimental observations of interest were changes in the localization and mobility of the nanoscale organization of synaptic molecules on primary cultured hippocampal neurons. In these experimental conditions, the sample size was calculated with a power factor of $0.6-0.8$ and $\alpha$ of $0.2-0.5$, which required sample sizes of $5-12$ cells per condition depending upon the SD of the sample. To account for variability between cultures, samples were chosen over 2-4 independent cultures. These values were obtained from the power and sample size calculator from statistical solutions. (Source: http://www.statisticalsolutions.net/ pss_calc.php).

Modeling. AMPAR kinetics were modeled using Monte Carlo simulation essentially as described previously (Glavinovic and Rabie, 1998; Franks et al., 2002; Raghavachari and Lisman, 2004; Heine et al., 2008) using a 7 state model (see Fig. 10) and published values for kinetics rate constants, adjusted to fit the experimentally recorded miniature EPSCs (mEPSCs) in our system. The basic model considered a $300 \times 300 \mathrm{~nm}$ postsynaptic membrane with 400 receptors disposed at varying distances. Glutamate (3000 molecules) was released in the presynaptic cell lying $15 \mathrm{~nm}$ away from the postsynapse. Glutamate was allowed to diffuse at $0.1 \mu \mathrm{m}^{2} / \mathrm{ms}$ (Nielsen et al., 2004; Budisantoso et al., 2012) as in the synaptic cleft (15 nm wide). Monte Carlo simulations were performed with a $\Delta \mathrm{T}=1 \mu$ s time step. For the kinetic constants, the values were modified from Heine et al. (2008): $\mathrm{C} 1$ to $\mathrm{C} 2, \mathrm{~K} 1=4,590,000 \mathrm{M}^{-1} \mathrm{~s}^{-1}$; $\mathrm{C} 2$ to $\mathrm{C} 1, \mathrm{~K} 1 \mathrm{r}=4260 \mathrm{~s}^{-1}$; $\mathrm{C} 2$ to $\mathrm{C} 3$, $\mathrm{K} 2=28,400,000 \mathrm{M}^{-1} \mathrm{~s}^{-1}$; $\mathrm{C} 3$ to $\mathrm{C} 2, \mathrm{~K} 2 \mathrm{r}=3260 \mathrm{~s}^{-1 ;} \mathrm{D} 7$ to D6, K3 = $1,270,000 \mathrm{M}^{-1} \mathrm{~s}^{-1}$; D6 to D7, K3r $=45.7 \mathrm{~s}^{-1} ; \mathrm{C} 3$ to O4, $\alpha=1000 \mathrm{~s}^{-1} ; \mathrm{C} 2$ to D7, $\alpha 1=2890 \mathrm{~s}^{-1}$; C3 to D6, $\alpha 2=120 \mathrm{~s}^{-1}$; O4 to D5, $\alpha 3=200 \mathrm{~s}^{-1}$; D6 to D5, $\alpha 4=16.8 \mathrm{~s}^{-1} ; 04$ to $\mathrm{C} 3, \beta=200 \mathrm{~s}^{-1}$; D7 to $\mathrm{C} 2, \beta 1=20 \mathrm{~s}^{-1}$; D6 to $\mathrm{C} 3, \beta 2=0.727 \mathrm{~s}^{-1}$; D5 to $04, \beta 3=4 \mathrm{~s}^{-1}$; and D5 to D6, $\beta 4=190 \mathrm{~s}^{-1}$.

\section{Results}

Visualization of AMPAR subunit organization by superresolution light microscopy and electron microscopy

We applied four super-resolution imaging techniques to identify AMPAR distribution and mobility at a resolution well below the diffraction limit of light microscopy. On living hippocampal neurons, we applied sptPALM (Manley et al., 2008) and UPAINT (Giannone et al., 2010) to obtain high-density maps of AMPAR localization and movement on neuronal surfaces. These techniques are based on the localization of individual fluorescent molecules, either by stochastic activation of isolated fluorescent molecules (PALM) or by stochastic labeling (uPAINT) of the molecule of interest to provide high-density localization of isolated single molecule events $\left(\sim 1000\right.$ localizations $\left./ \mu \mathrm{m}^{2} / \mathrm{min}\right)$ over neuronal surfaces at a spatial resolution $\sim 45 \mathrm{~nm}$. Third, STED microscopy (Hell, 2009) was used as an additional bulk super-resolution imaging technique to investigate the nanoscale AMPAR localization in fixed hippocampal neurons. Fourth, dSTORM (Heilemann et al., 2008; van de Linde et al., 2011), also called Ground State Depletion followed by Individual Molecule return (Folling et al., 2008), was used as a dual-color single molecule-based super-resolution microscopy method to describe the respective nanoscale distribution of AMPARs and PSD95 at a resolution of $\sim 20 \mathrm{~nm}$. Finally, we compared these superresolution light microscopy data with AMPAR distribution observed by pre-embedding immunogold EM.

\section{sptPALM revealed that AMPARs are confined in stable subsynaptic clusters}

sptPALM experiments were performed on neurons transfected with GluA1 and GluA2 AMPAR subunits genetically fused with the variant of Eos photo-switchable protein, mEos2 (referred to as Eos::GluA1 and Eos::GluA2). Homer1c fused to the Cerulean fluorescent protein (Homer1c::Cerulean) was used as a synaptic marker because it labels postsynaptic sites and does not interfere with the mobility and localization of AMPARs (Bats et al., 2007).

Diffraction limited epifluorescence images of Eos::GluA1 displayed a relatively homogeneous distribution of GluA1 proteins along the dendrites and enrichment in the spine head (Fig. 1A; Epifluorescence). In contrast, sptPALM superresolved intensity images obtained from living neurons presented a strongly nonuniform distribution of Eos::GluA1 in different subcellular regions (Fig. $1 A$; sptPALM-Intensity). Reconnecting the localization of individual Eos::GluA1 molecules between sequential images allowed us to compute the trajectories of individual GluA1 molecules along the neuronal surface (Fig. $1 A$; sptPALM-Trajectories) and to map the instantaneous diffusion coefficient distribution (Fig. 1A; sptPALM- 


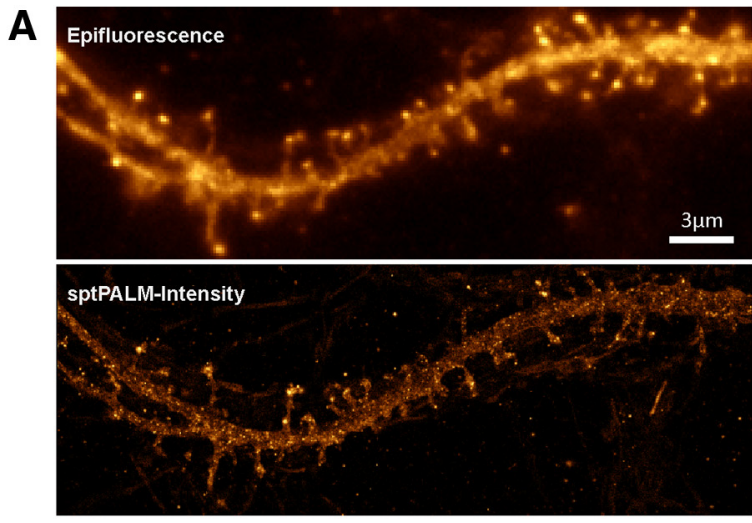

B
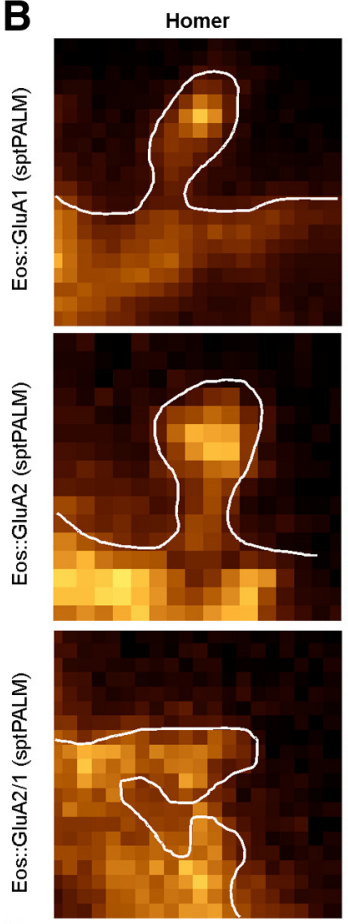

D

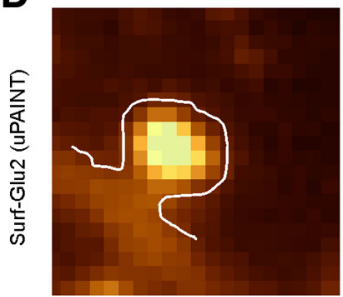

Intensity map
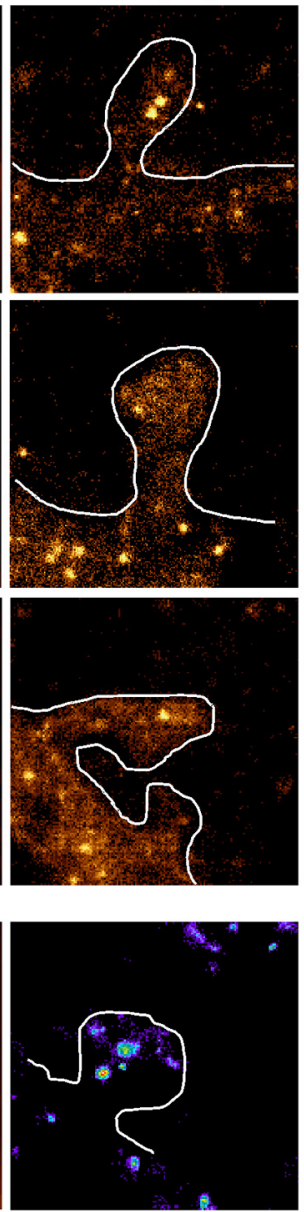
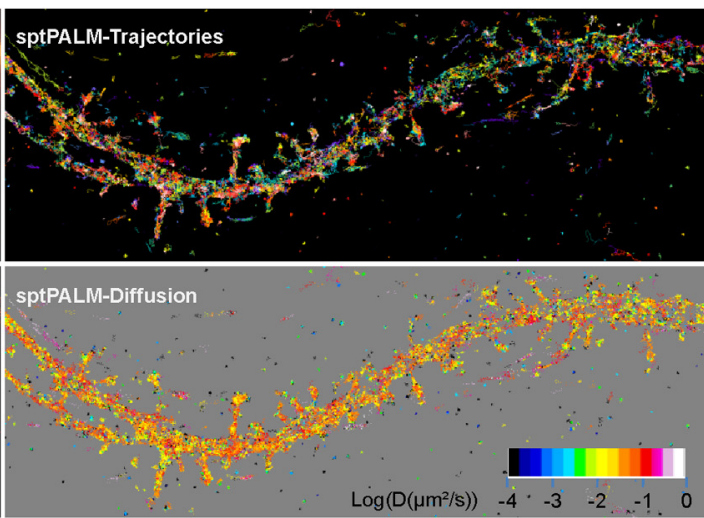

Trajectories
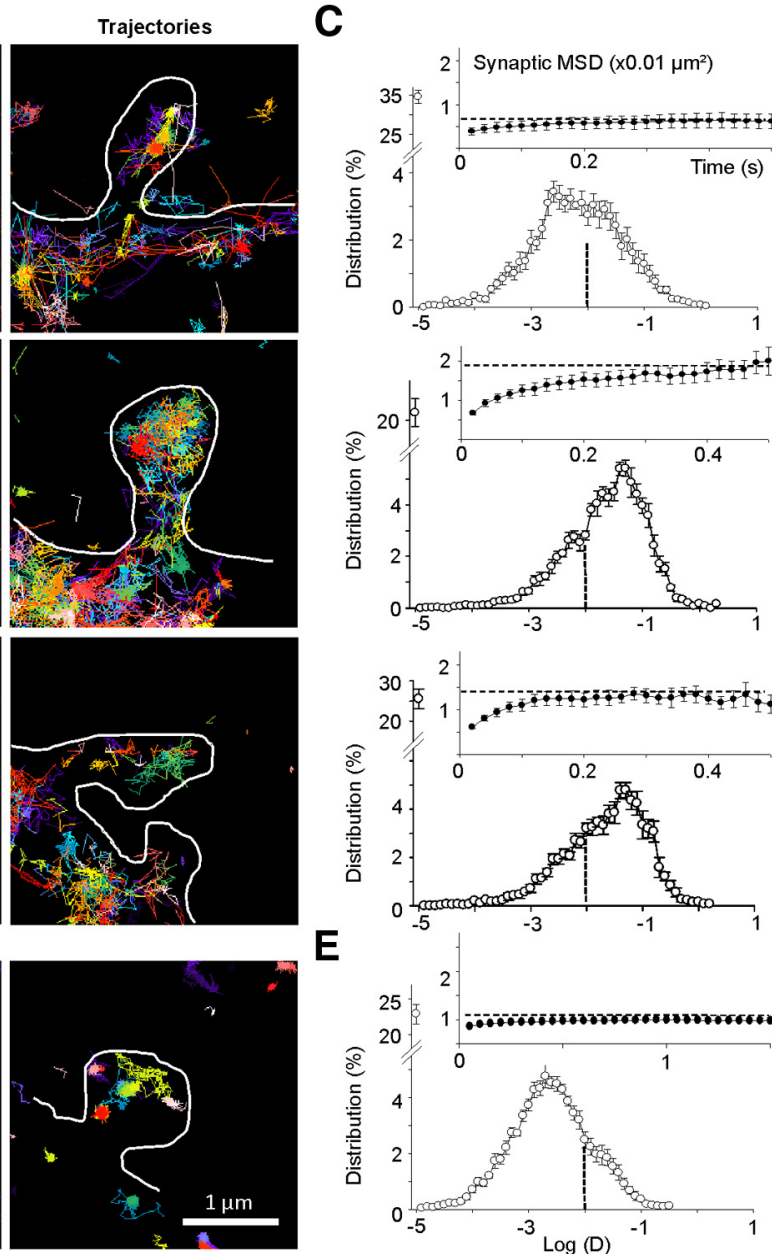

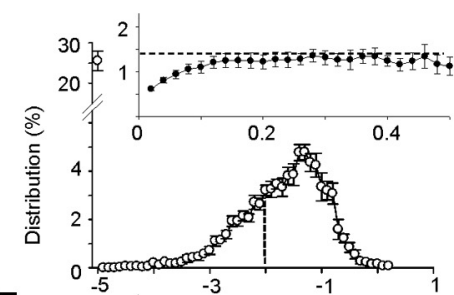

E

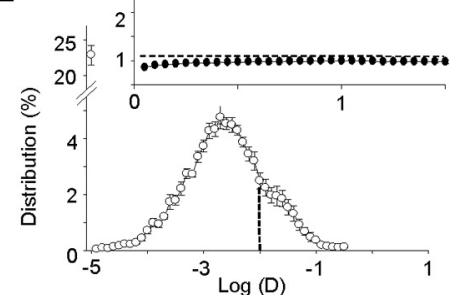

Figure 1. High-density and super-resolution light imaging of AMPAR localization reveals organization in nanodomains. $A$, Comparison between epifluorescence, super-resolution intensity image, trajectories, and diffusion map of a neuron expressing Eos::GluA1. The epifluorescence image is obtained from the native nonphotoconverted green form of Eos molecule. The super-resolution intensity image is obtained by sptPALM from a sequence of 20,000 images of sparse single molecules in the photoconverted Eos red channel. The images of trajectories represent the individual trajectories longer than 8 frames ( $20 \mathrm{~ms}$ per frame) of activated single Eos molecules. The diffusion map corresponds to the average instantaneous diffusion coefficients computed from the MSD of each trajectory. $\boldsymbol{B}, \boldsymbol{D}$, Examples of AMPAR organization inside spines of live hippocampal neurons by sptPALM (B) and uPAINT (D). Synapses are labeled with the expression of either Homer1c::Cerulean for sptPALM experiments or Homer1c::GFP for uPAINT. From left to right, Epifluorescence images of expressed Homer1c, the corresponding super-resolution sptPALM intensity images and trajectories obtained from cells transfected with Eos::GluA1, Eos::GluA2, Eos::GluA2 plus untagged GluA1, or uPAINT of endogenously expressed GluA2 containing AMPAR (Surf-GluA2). C, E, Average distribution of instantaneous diffusion coefficients and MSD curves (insets) for sptPALM ( $C$ ) and UPAINT $(\boldsymbol{E})$ of synaptic AMPAR. Error bars indicate cell-to-cell variability. For sptPALM experiments, the peak of the MSD distribution for E0s::GluA1 is left shifted compared with that of Eos::GluA2, indicating a larger fraction of slowly mobile versus highly mobile E0s::GluA2-containing receptors (threshold indicated by the vertical dashed line). Coexpression of untagged GluA1 with Eos::GluA2 partially restores AMPAR organization into nanodomains and the immobilization of Eos::GluA2-containing receptors. For uPAINT experiments, the MSD histogram for Surf-GluA2 is shifted toward the immobile fraction but indicates a bimodal distribution similar to overexpressed Eos::GluA1. E0s::GluA1 monitored by sptPALM and Surf-GluA2 receptors recorded with UPAINT present a high confinement inside synapses compared with Eos::GluA2 monitored by sptPALM. The confinement area obtained with the MSD plateau (horizontal dashed line) is 4-5 times larger for Eos::GluA2 than Eos::GluA1 and Surf-GluA2. This indicates that Eos::GluA2 AMPAR explore more surface area than Eos::GluA1or endogenous Surf-GluA2 AMPAR in spines. 
Diffusion). We could observe several thousand trajectories in a single dendritic segment within 1-2 min. This coarse cartography revealed a high heterogeneity of receptor mobility between subcellular regions. To ensure the surface expression of Eos-tagged receptors, we incubated neurons expressing a GluA1 construct with a TEV cleavage site between Eos and the GluA1 N terminus (Eos::TEV::GluA1) with the active TEV protease. This resulted in a $90-95 \%$ reduction in single molecule fluorescence (Fig. 2A), indicating that most Eos::TEV::GluA1 fluorescence was on the cell surface.

We used sptPALM data to compute the localization and trajectories of AMPARs (Figs. $1 B$ and $2 E$ ) as well as the distribution of receptor mobility inside synaptic sites labeled by Homer1c::Cerulean (Fig. 1C) and compared the differences in global mobility of Eos::GluA1, Eos::GluA2 (Fig. 2E), and surface native AMPARs (Fig. $2 F$ ). Overall, the mobility of overexpressed AMPAR and endogenous surface receptors was faster in the dendritic shafts than in synaptic areas (Fig. 2E), as previously shown (Bats et al., 2007). Close examination of super-resolved intensity images and trajectory maps of Eos::GluA1 at spines indicated that Eos::GluA1 is concentrated in several distinct clusters of sizes smaller than the diffraction limit (Fig. $1 B$; GluA1-Intensity map) and that over half of Eos::GluA1 moves $<0.01 \mu \mathrm{m}^{2} / \mathrm{s}$ inside synapses (Fig. 1B; GluA1-Trajectories; and Fig. 1C; Distribution). Within these clusters, the receptors undergo confined movements, as indicated by the plateau reached by the mean square displacement (MSD) curve (Fig. $1 C$; MSD plateau $\sim 0.006 \mu \mathrm{m}^{2}$, median values of the diffusion coefficient $D$ in $\mu \mathrm{m}^{2} / \mathrm{s}$ with interquartile range [IQR] for Eos::GluA1; dendrites $0.0153 \mathrm{IQR}$ $0.00036-0.0687$, synapses 0.0032 IQR $0.00001-0.0195)$. In contrast, expression of Eos::GluA2 yielded different images, the formation of synaptic clusters being largely reduced (Figure $1 B$; GluA2-Intensity map). Eos::GluA2 moved faster (Fig. 1C; GluA2 Distribution) and trajectories (Fig. $1 B$; GluA2-Trajectories) displayed higher diffusion and much less confinement (Fig. $1 C$; MSD, plateau $\sim 0.02 \mu \mathrm{m}^{2}$, diffusion coefficient of Eos::GluA2 in $\mu \mathrm{m}^{2} / \mathrm{s}$; dendrites 0.052 IQR $0.014-0.127$, synapses 0.015 IQR 0.001-0.052). This indicates that Eos::GluA2 visits 4 times more synaptic surface than Eos::GluA1 in the same time. For Eos::GluA2-expressing cells, $>90 \%$ of the spines did not exhibit synaptic clusters. Expression of individual subunits mostly forms homomers, whereas endogenous receptors are mostly formed of GluA1/GluA2 heteromers. Interestingly, the coexpression of Eos::GluA2 with nontagged GluA1 partially rescued the formation of subsynaptic clusters (Fig. 1B; GluA1/2-Intensity map). Cells coexpressing Eos::GluA2 and GluA1 displayed an increased confinement of Eos::GluA2 and a decreased diffusion (Fig. 1C; GluA1/2 MSD, plateau $\sim 0.013 \mu \mathrm{m}^{2}$, diffusion coefficient of Eos::GluA2+GluA1 in $\mu \mathrm{m}^{2} / \mathrm{s}$; dendrites 0.032 IQR 0.003-0.103, synapses 0.0138 IQR $0.0002-0.056$ ). This was also consistent with changes in the global mobility of Eos::GluA2 where the fraction of Eos::GluA2 moved slower when coexpressed with untagged GluA1 (Fig. 2E).

Together, these data suggest that expressed GluA1 subunits are less mobile and are organized into subdiffraction sized clusters on hippocampal spines compared with ectopic GluA2 subunits. However, the subdiffraction limited organization and the slow moving fraction of receptors can be partially restored on ectopic GluA2 when coexpressing GluA2 with GluA1. This indicates the importance of GluA1 subunit in the subspine organization and retention of AMPARs on the synaptic membrane.

\section{uPAINT revealed that AMPARs are confined in stable subsynaptic clusters}

uPAINT was performed as a complementary high-density single particle tracking technique to monitor the localization and movement of endogenous AMPARs located on the neuronal surface plasma membrane. We used a monoclonal antibody directed against an extracellular region of the GluA2 AMPAR subunit conjugated to the ATTO647N fluorescent dye to randomly label surface AMPARs containing the GluA2 subunit on living hippocampal neurons (Fig. 1D; Surf-GluA2). This method specifically labeled endogenous GluA2 containing AMPARs as demonstrated by the loss of labeling observed in neurons cultured from GluA2 KO mice (Fig. 2B). Furthermore, it provided information on the general behavior of endogenous AMPARs because the majority of AMPARs are either GluA2/GluA1 or GluA2/GluA3 heterotetramers (Wenthold et al., 1996). In contrast to sptPALM, uPAINT is a technique based on specific stochastic labeling (Fig. 2C) that does not allow for the labeling of all available receptors on the surface. This drawback is counterbalanced by the advantage that no overexpression is needed and that each antibody is labeled with several organic fluorophores, providing a bright probe that can be localized with better accuracy and observed for longer durations than Eos molecules.

uPAINT of GluA2-containing AMPARs yielded a strongly nonuniform distribution of fluorescence intensity (Fig. 1D; SurfGluA2-Intensity map) similar to that obtained during sptPALM images of Eos::GluA1-containing receptors. In each spine, AMPARs were concentrated in a few subdiffraction-sized clusters. Receptors were mainly slowly diffusing and highly confined in these domains (Figure 1D; Trajectories; and Fig. 1E; MSD; plateau $\sim 0.006 \mu \mathrm{m}^{2}$, diffusion coefficient of Surf GluA2 in $\mu \mathrm{m}^{2} / \mathrm{s}$; dendrites 0.0012 IQR 0.00001-0.007, synapses 0.0008 IQR 0.00001-0.0048). As previously reported (Bats et al., 2007; Opazo et al., 2010), endogenous GluA2-containing receptors moved slower than overexpressed subunits. Together, both sptPALM of expressed Eos::GluA1 homomers or Eos::GluA2/untagged GluA1 heteromers and uPAINT of endogenous GluA2-containing AMPARs yielded a comparable distribution of receptors confined in subsynaptic clusters.

\section{STED confirmed that AMPARs are concentrated in subsynaptic clusters}

The intensity localization maps of AMPARs reconstructed from sptPALM and UPAINT imaging experiments rely on the accumulation of multiple sequential single molecule localizations and may contain undersampling biases. We thus acquired images of AMPAR distribution using the alternative STED nanoscopic imaging approach (Hell, 2009). We live-stained neurons expressing the synaptic marker Homer1c::GFP with the same monoclonal antibody against an extracellular epitope of GluA2 used in uPAINT, followed by a secondary antibody coupled to ATTO647N. Postsynapses labeled with Homer1c::GFP were first identified by scanning confocal microscopy (Fig. 3A; Confocal Homer1c). STED images of the surface localized endogenous GluA2 were then acquired (Fig. 3A; STED GluA2). Consistent with sptPALM and uPAINT experiments, STED images confirmed that AMPARs are highly clustered into subdiffraction sized domains at synapses (Fig. 3B; sptPALM, uPAINT, STED).

\section{Subsynaptic AMPAR clusters are nanometer-scale "nanodomains"}

The super-resolution techniques used in this study enabled the subdiffraction size and shape of AMPAR clusters to be measured. 

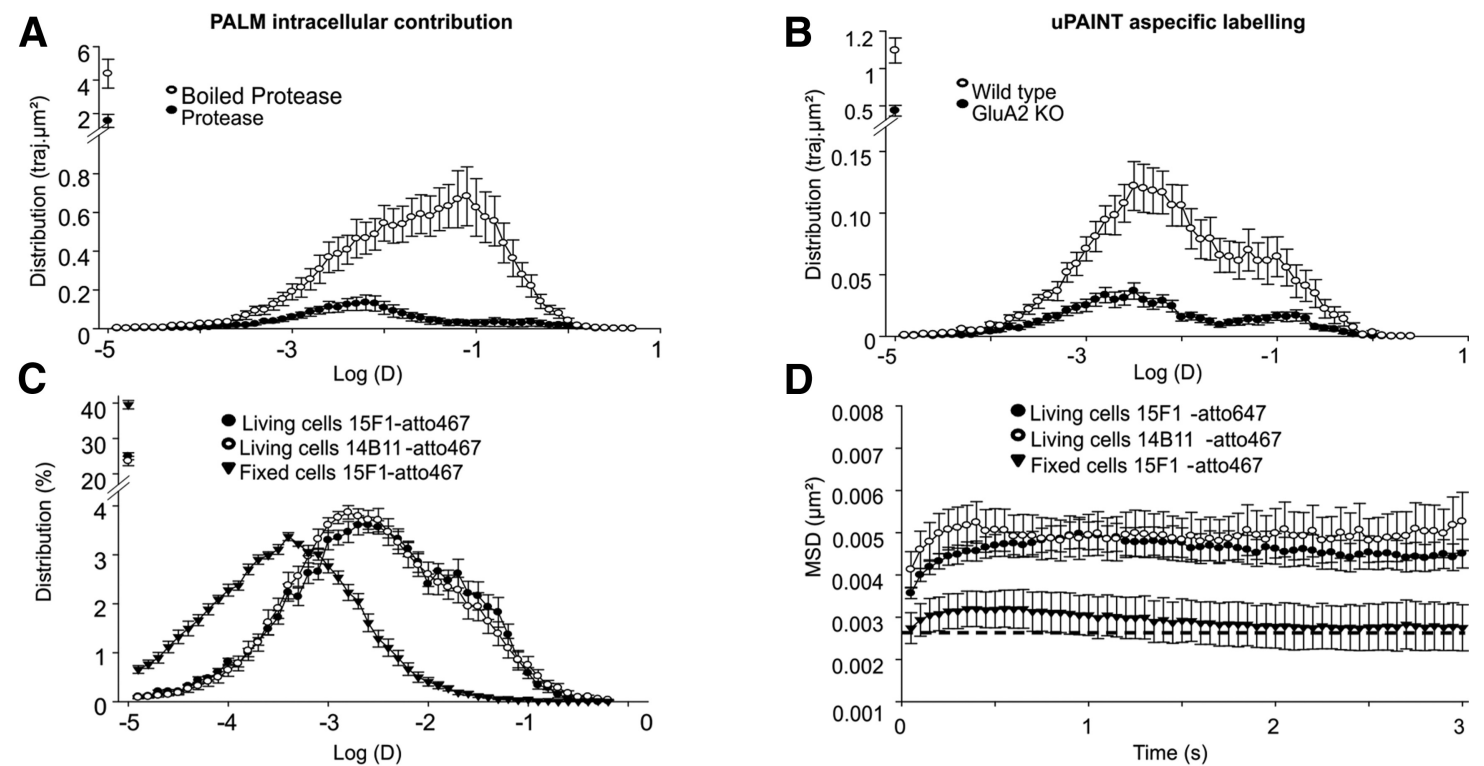

\section{E}
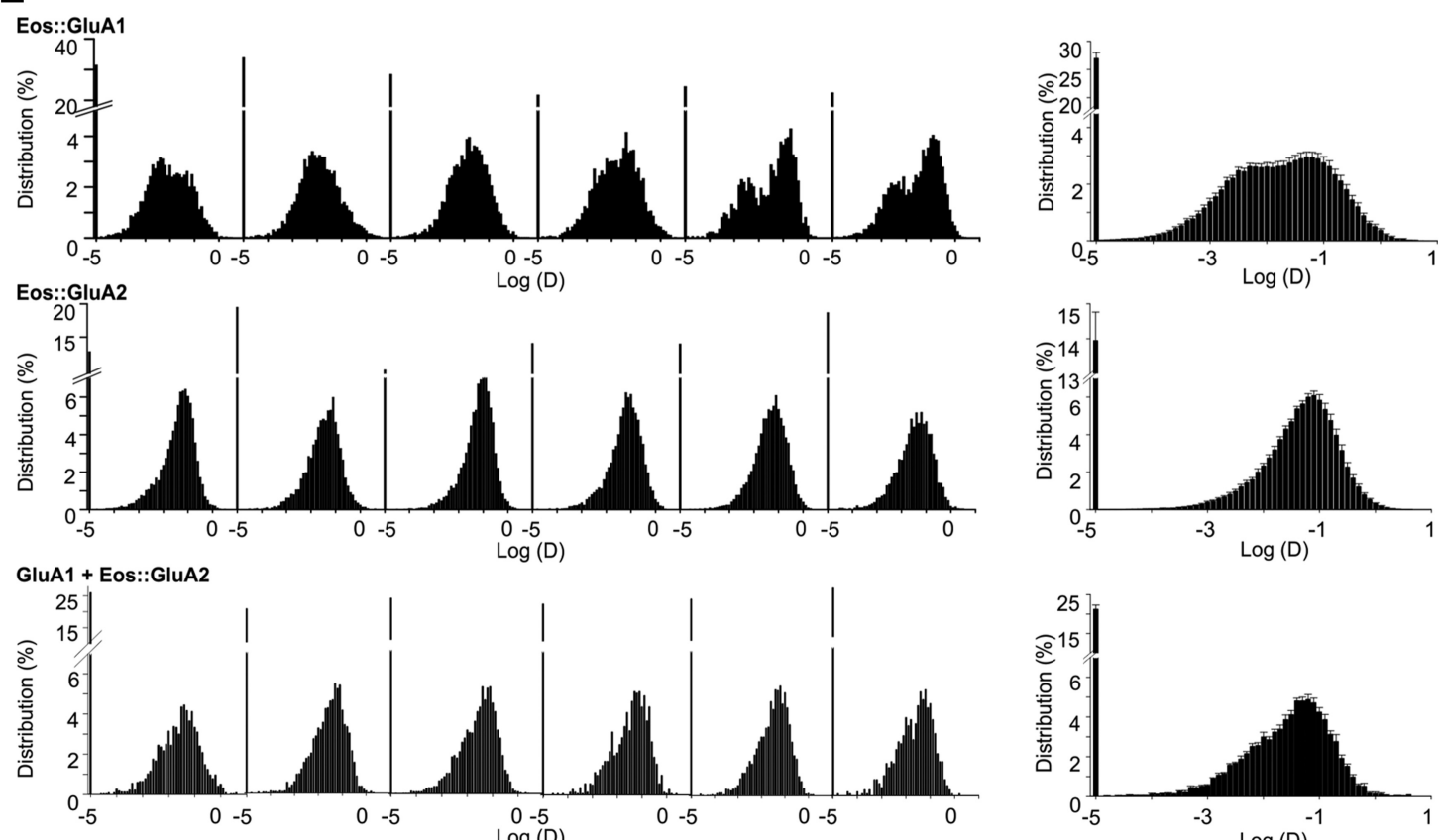

$\mathbf{F}$
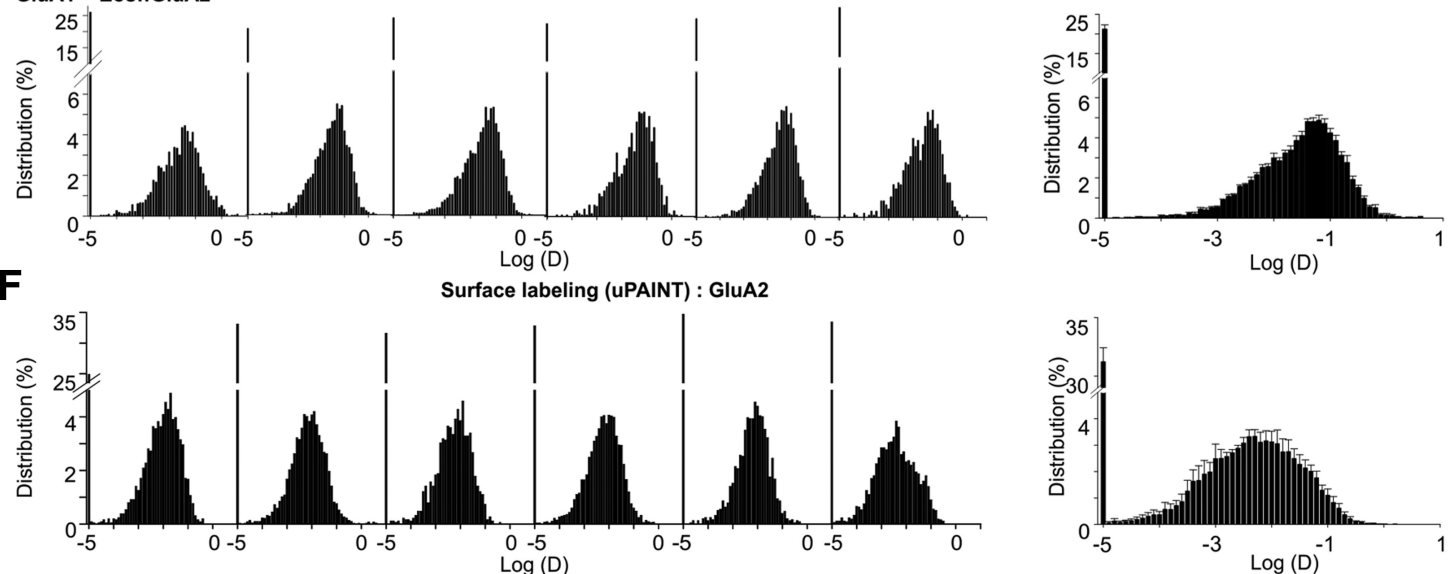

Figure 2. Comparativecharacteristics of endogenous and overexpressed AMPAR diffusion in live and fixed neurons. $A$, Specificity of sptPALM localization. Contribution of the intracellular staining to the signal detected in sptPALM images was analyzed by comparing the histograms of instantaneous diffusion coefficients on cells where extracellular Eos coupled to GluA1 was cleaved by the TEV protease (protease) to that of control cells incubated with protease inactivated by boiling (boiled protease). B, Specificity of the antibodies binding in uPAINT. Comparison of the intensity of GluA2 endogenous labeling with uPAINT technique on wild-type neurons (wild-type) and neurons from GluA2 knock-out mice. These experiments reveal a nonspecific labeling of $\sim 15 \%$. Instantaneous diffusion histograms are normalized to trajectories/ $\mu \mathrm{m}^{2}$.C, Comparison of AMPAR mobility in live and fixed cells with different antibodies. Two different antibodies yield comparable mobility histograms in live cells. Performing uPAINT on fixed cells results in a histogram shifted toward immobile fractions. $\boldsymbol{D}$, Different antibodies against GluA2 used for uPAINT provide comparable confinement kinetics. The confinement value of fixed molecules is twofold smaller than the confined fraction of mobile AMPAR in the synapse, indicating that the accuracy of localization does not limit the measurement of nanodomain confinement properties in live neurons. $E, F$, Diffusion coefficient histograms of various AMPAR subunits in multiple cells using sptPALM $(\boldsymbol{E})$ and uPAINT $(\boldsymbol{F})$. Illustration of the variability of instantaneous diffusion coefficient histograms from whole dendritic segments of neurons transfected with Eos::GluA1, Eos::GluA2, Eos::GluA2 + untagged GluA1, and endogenous surface AMPAR. For each construct, the distribution of six different cells is provided together with the mean distribution computed from all cells (rightmost histograms). Eos::GluA2 mobility distribution presents a narrow distribution enriched in highly mobile fraction, whereas Eos::GluA1 shows a broad bimodal distribution with a fast and slow or immobile fraction. Surface AMPA receptors labeled by antibody against GluA2 exhibit a broad distribution similar to that of Eos::GluA1. 


\section{A}

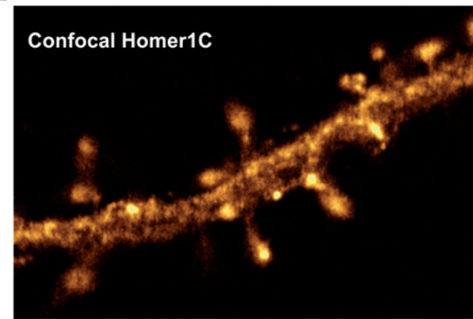

B

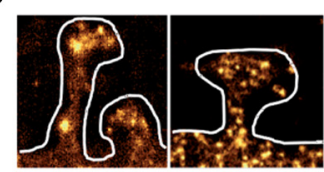

C

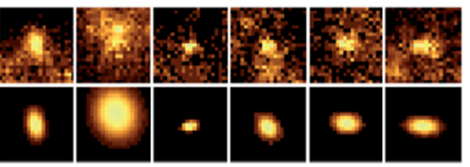

D

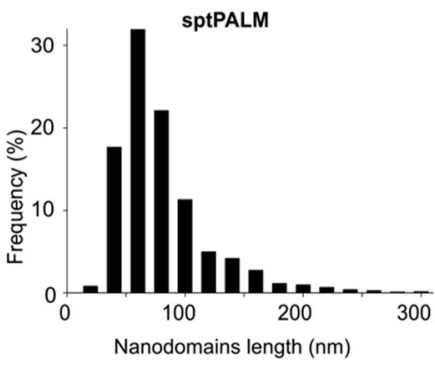

E

Principal Axis

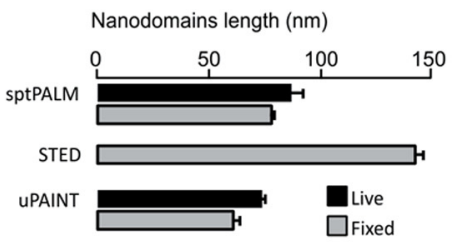

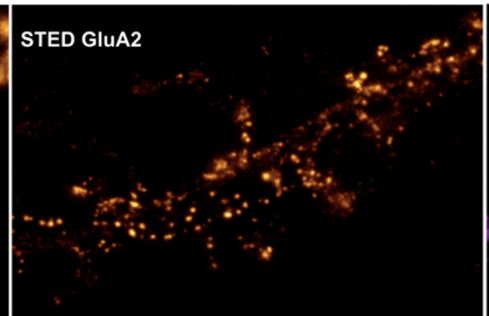

STED
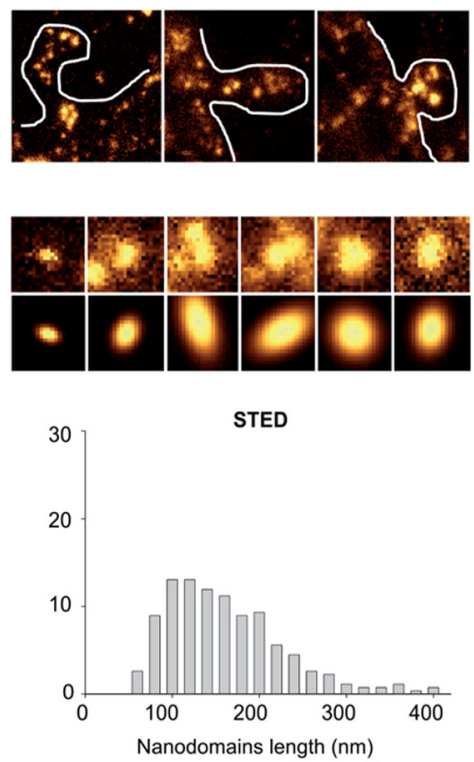

Auxiliary Axis

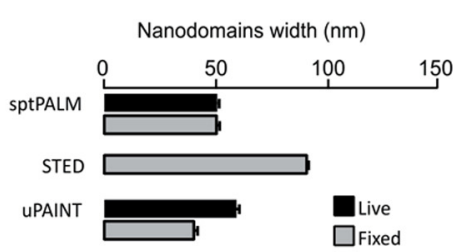

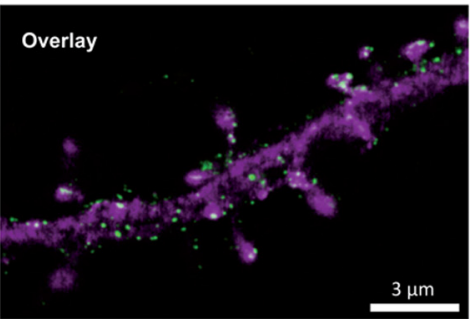

UPAINT
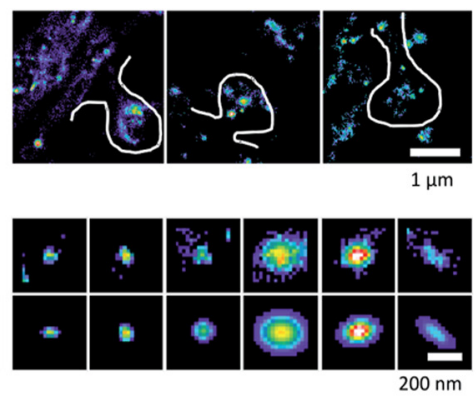

uPAINT

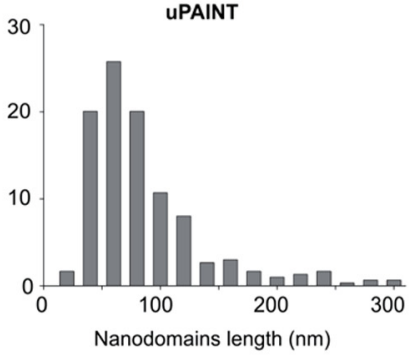

$\mathbf{F}$

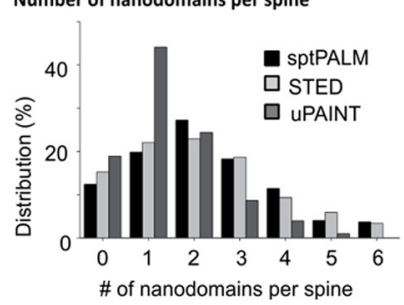

Figure 3. Quantification of AMPAR nanodomain size and density by multiple super-resolution light imaging techniques. A, Confocal and STED images of a dendritic segment expressing Homer1c::GFP (left, confocal) that was live-stained for Surf-GluA2-ATT0647N for STED imaging (middle, STED). Overlaid images (right; Homer1c::GFP shown in magenta; STED GluA2 shown in green). B, Gallery of super-resolution images of spines imaged by sptPALM (Eos::GluA1), STED, and uPAINT (Surf-GluA2-ATT0647N). C, Examples of AMPAR nanodomains imaged by sptPALM, STED, and uPAINT, and the corresponding fit using 2D anisotropic Gaussian functions. D, Distributions of the principal (length) axis of nanodomains obtained by 2D anisotropic Gaussian fitting. sptPALM, STED, and uPAINT display similar distributions centered at 80,120 , and $70 \mathrm{~nm}$, respectively. $E$, Comparison of the mean lengths (principal) and widths (auxiliary) of nanodomains in living and fixed cells for the different techniques. $F$, Quantification of the number of nanodomains per spine with the different imaging techniques shows that $\sim 50 \%$ of spines contain one or two nanodomains.

Anisotropic bidimensional Gaussian fitting was applied (Fig. 3C) as described in Materials and Methods (clusters contained in $n=$ 323 synapses by sptPALM, 126 by uPAINT, 118 by STED). The long (principal) and short (auxiliary) axes were determined as the full width at half-maximum (FWHM) of the fitted Gaussian. Most of the principal axes were measured to be smaller than the diffraction limit, with a median of $69.6 \mathrm{~nm}$ (IQR 54-93 nm) by sptPALM, $78.1 \mathrm{~nm}$ (IQR 64.9-87.1 nm) by uPAINT, and $132 \mathrm{~nm}$ (IQR 98-191 nm) by STED (Fig. 3D). These values are in accordance with the small size of confinement obtained from MSD curves (Fig. 1C). Because of their nanoscale dimensions, we henceforth refer to these AMPAR clusters detected by superresolution microscopy as AMPAR nanodomains.

Most AMPAR nanodomains exhibited an ovoid shape with an average shape factor of $0.6-0.7$ (Fig. $3 E$ ). As a control, we performed similar nanodomain quantifications on fixed samples.
The principal axes of AMPAR nanodomains in fixed samples were distributed around a median of $78.1 \mathrm{~nm}$ (IQR 54-95.7 nm) and $62.8 \mathrm{~nm}$ (IQR $48.9-80.3 \mathrm{~nm}$ ) using sptPALM and uPAINT, respectively, similar to that obtained in live-cell experiments (Fig. $3 E)$. The confinement area of AMPAR movements measured by uPAINT experiments on fixed neurons $\left(0.0028 \mu \mathrm{m}^{2}\right)$ is almost twofold smaller than the average size of the nanodomains $(0.005$ $\mu \mathrm{m}^{2}$ ) (Fig. $2 D$ ), indicating that the determination of the nanodomain size is not limited by the pointing accuracy. Similarly, the resolution provided by sptPALM under our experimental conditions was better than $50 \mathrm{~nm}$ (see Materials and Methods). The larger nanodomain size measured by STED likely reflects its poorer resolution in our acquisition conditions.

Finally, we quantified the fraction of AMPARs in nanodomains and the number of nanodomains per spine. All imaging techniques showed that $\sim 65 \%$ of the receptors were concen- 

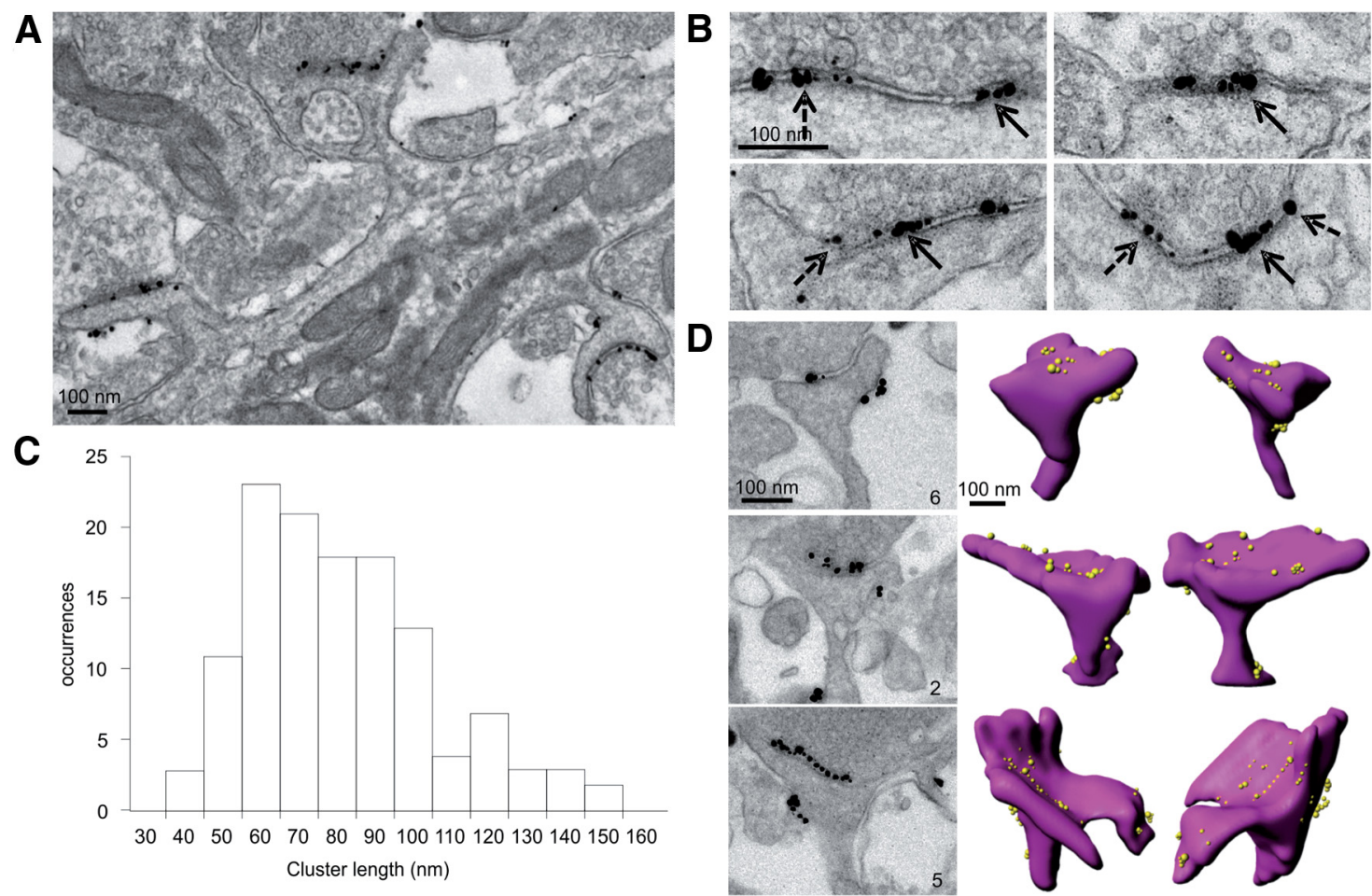

Figure 4. Nanodomain size and per-spine abundance revealed by pre-embedding immunogold EM. A, Low-magnification view of a portion of dendrite containing three synaptic spines that was live-labeled for GluA2 before fixation and then labeled with secondary fab' fragment conjugated to a $1.4 \mathrm{~nm}$ gold particle. Silver-intensified label particles are highly concentrated at PSD-membrane in spines $(7.23$ particles $/ \mu \mathrm{m})$. Label particles are also present on extrasynaptic spine membrane (2.12 particles $/ \mu \mathrm{m})$ and at a relatively low density on the dendritic plasma membrane (1.24 particles $/ \mu \mathrm{m}$ ). $\boldsymbol{B}$, High-magnification views of individual spine synapses show that label particles are present in nanodomains (solid arrows) or as single-label particles (dashed arrows). $C$, Histogram of the clusters of immunogold particles size in $2 D$ EM sections showing that the average length of clusters of two or more label particles fits a single Gaussian with a mean of $77.48 \pm 23.98$. D, Images of three spines that were serial sectioned and used for $3 \mathrm{D}$ reconstructions (right) and count the number of nanodomains per spine. The number in lower right corner of each EM image indicates the number of nanodomains counted on each spine.

trated in nanodomains and that $>80 \%$ of spines contained at least one nanodomain ( $88 \%$ by sptPALM, $82 \%$ by uPAINT, and $85 \%$ by STED) (Fig. $3 F$ ). The average number of nanodomains per spine was $\sim 2.5(2.42 \pm 0.18, n=584$ spines $)$ when measured with sptPALM. STED yielded similar results $(2.38 \pm 0.17, n=$ 351 spines), whereas uPAINT led to a lower average of $1.4 \pm 0.1$ ( $n=299$ spines), probably because of the intrinsic underlabeling of the technique (Fig. $3 F$ ). Spines without nanodomains usually exhibited a low level of diffuse AMPAR staining that was not further quantified.

\section{AMPAR nanodomains detected by live-labeling and pre-embedding immunogold electron microscopy}

Despite the advent of super-resolution optical microscopy, immunogold labeling by EM still provides the highest resolution for AMPAR localization and remains the "gold standard" (Kharazia et al., 1996; Bernard et al., 1997; Kharazia and Weinberg, 1997; Nusser et al., 1998; Takumi et al., 1999b; Ganeshina et al., 2004), with the important drawback that it can only image fixed tissue. Most previous EM studies have used the postembedding immunolabeling method, which provides incomplete information about the distributions of receptors because of its relatively low sensitivity (Nusser et al., 1998; Masugi-Tokita and Shigemoto, 2007). Pre-embedding immunogold labeling (Bernard et al., 1997) allows mapping the distribution of surface AMPARs with increased sensitivity and has been recently used to observe the distribution of GluA2, the most prevalent subunit in AMPARs (Tao-Cheng et al., 2011). Here, we performed similar preembedding immunogold labeling, with two deviations. First, we immunolabeled surface GluA2-containing AMPARs in live neurons in contrast to the common postfixation protocols. Second, instead of the commercial 6C4 anti-GluA2 clone, we used a GluA2 antibody with excellent avidity, as confirmed by uPAINT experiments (Fig. 2B) (Giannone et al., 2010). After 3 min of live-labeling, neurons were fixed, labeled with a secondary fab' fragment coupled to nanogold ( $1.4 \mathrm{~nm}$ size), and processed for EM including silver enhancement of nanogold as indicated in Materials and Methods.

A typical view of surface labeling is shown in Figure $4 A$. The density of immunogold labeling at the PSD membrane in spines was 7.23 particles/ $\mu \mathrm{m}$. This was $\sim 3.5$ times more dense than labeling on extrasynaptic spine membrane (2.12 particles/ $\mu \mathrm{m})$ and 5 times more concentrated than along the dendritic shaft membrane ( 1.24 particles $/ \mu \mathrm{m})(n=538$ label particles measured on $31.94 \mu \mathrm{m}$ of PSD membrane, $74.59 \mu \mathrm{m}$ of extrasynaptic spine membrane and $120.65 \mu \mathrm{m}$ of dendritic shaft membrane). Interestingly, immunolabel was often organized in clusters located in the PSD as well as on extrasynaptic membranes (Fig. 4B). We measured the size of the immunolabel clusters (defined as 2 or more particles separated by $<20 \mathrm{~nm}$ ) (Fig. $4 C$ ) and found that the average cluster length was $77.48 \pm 23.98 \mathrm{~nm}(n=123$ clusters). This size is in close agreement with the size of nanodomains measured by the super-resolution light microscopy techniques. The size of particle clusters did not vary between membrane regions (PSD clusters $=81.48 \pm 27.12 \mathrm{~nm}$; spine clusters = $72.72 \pm 20.04 \mathrm{~nm}$; dendritic shaft clusters $=77.04 \pm 23.32 \mathrm{~nm}$ ), and each cluster contained on average $\sim 3$ label particles. Similar to findings at the super-resolution level, $\sim 60 \%$ of the label was 
A
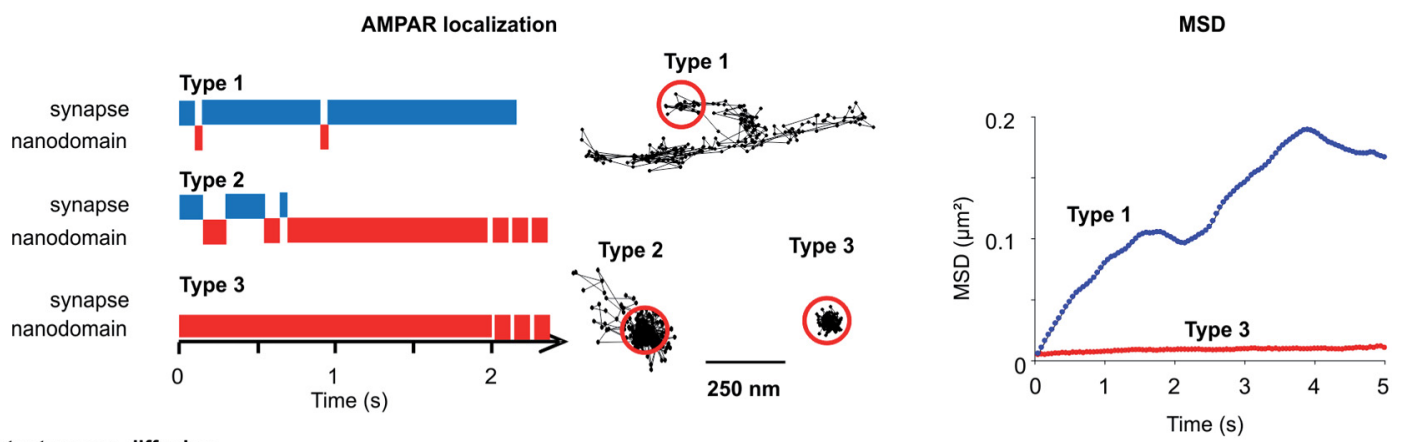

B Instantaneous diffusion
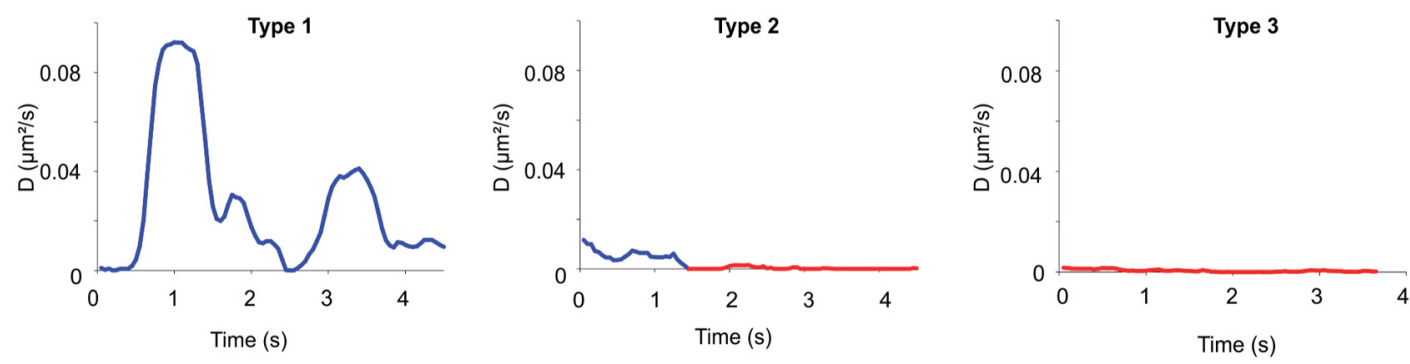

C
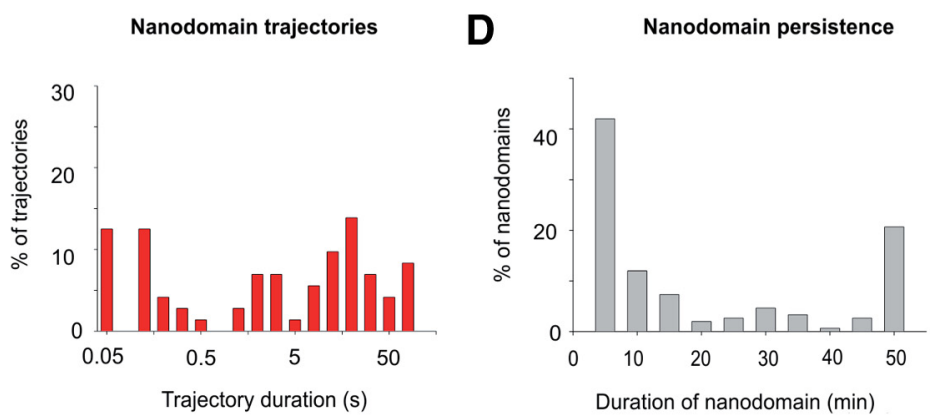

Figure 5. Dynamics of AMPAR nanodomain composition, shape, and position. $A$, Illustration of the diffusive behavior of Surf-GluA2-ATT0647N-labeled endogenous AMPAR inside and outside nanodomains observed by uPAINT. Measurement of the dynamics of AMPAR localization illustrates three different types of behavior within and in between nanodomains. The times spent by individual AMPAR inside and outside nanodomains are represented in red and blue, respectively. In Type 1, AMPAR randomly diffuse in the vicinity of nanodomains without getting trapped. In Type 2, AMPAR alternate between inside and outside the nanodomains before getting strongly trapped in the nanodomain and confined. In Type 3, AMPAR are immobilized for the duration of the recording in the nanodomain. Examples of trajectories for the three types of behaviors are displayed, with red circles indicating the nanodomains. MSD curves of Type 1 (blue) and Type 3 (red) illustrate a strong confinement of Type 3 molecules compared with Type 1. B. Graph of the instantaneous diffusion coefficient versus time that reveal a slow AMPAR diffusion inside nanodomains and a much faster one in synapse but outside of nanodomains. C, Duration of synaptic single-molecule trajectories inside and outside nanodomains. Synaptic trajectories outside nanodomain display a residency time of $<500 \mathrm{~ms}$ for $>90 \%$ of the observed trajectories. For intrananodomain molecules, the residency time is longer than $5 \mathrm{~s}$ for at least $50 \%$ of the trajectories, confirming the AMPAR retention inside nanodomains. $\boldsymbol{D}$, Nanodomain stability histogram measured using time-lapse sptPALM shows that $>20 \%$ of the nanodomains remain stable during the entire experiment duration (almost $1 \mathrm{~h}$ ).

contained in a cluster, a value that was consistent between membrane regions (\% of particles in cluster: PSD 55\%, spine $60 \%$, dendritic shaft $66 \%$ ). Based on these similarities, we concluded that the clustered immunolabel viewed at the EM level corresponds to nanodomains detected using super-resolution light microscopy.

To count the number of nanodomains present on individual spines and specify their location in the PSD or extrasynaptic spine membrane, seven spines were reconstructed by serial section EM (Fig. 4D). On average, there were $2.14 \pm 0.90$ nanodomains in the PSD and $4.14 \pm 1.86$ nanodomains on the spine extrasynaptic membrane.

Evolution and stability of AMPAR nanodomains in spines We investigated the dynamics of AMPARs between nanodomains by uPAINT because this approach provides the longest observation time of individual fluorescent probes (median recording time for a single anti-GluA2-ATTO647N conjugate =
$150 \mathrm{~ms}$, IQR $100 / 300, n=15000$, with $>10 \%$ of trajectories $>0.5$ $\mathrm{s})$. Single receptor trajectories could be classified into three distinct patterns (Fig. 5A). In the first pattern, molecules diffused in and out of nanodomains with random variations in the instantaneous diffusion coefficient that were uncorrelated to a specific identified localization of the protein. This suggested that the receptors did not engage in stabilizing interactions (Fig. $5 A$, B; Type $1)$. In the second pattern, receptors alternated between nanodomains and the rest of the spine space, displaying clear periods of confinement when colocalized with the nanodomains (Fig. 5A, $B$; Type 2). This was particularly apparent on the plot of the instantaneous diffusion coefficient versus time of these molecules that displayed a drastically reduced diffusion coefficient once the molecule localized in the nanodomain. Finally, a third group of molecules were observed to remain confined in the nanodomains with very low diffusion coefficients for several minutes, indicating a strong association with molecular components of the nanodomain (Fig. 5A, B; Type 3). The much stronger confinement of 
receptors in nanodomains was also apparent when comparing the average MSD for the type 1 and type 3 mobility modes (Fig. $5 A$; MSD). These plots also indicate that, in between nanodomains, the receptors undergo rapid free diffusion. Based on this observation, synapses were divided into two functional zones discriminated by receptor mobility: zones inside nanodomains and zones outside nanodomains. Interestingly, the residence time of receptors outside nanodomains was short while they randomly moved rapidly (median residence in extra-nanodomain $=$ $0.25 \mathrm{~s}$, IQR $0.1 / 0.7, n=161$; median total trajectory length $0.75 \mathrm{~s}$, IQR $0.55 / 1.4, n=5000)$. In contrast, molecules were confined inside nanodomains for much longer durations, going up to few minutes (median residence inside nanodomains $=2.25 \mathrm{~s}, \mathrm{IQR}$ $0.1 / 16.2, n=155$; Fig. $5 C$ ). Together, these data indicate that, inside synapses, AMPARs diffuse freely between nanodomains then rapidly hop inside nanodomains where they are strongly immobilized and reside for long periods. These stabilization domains could be related to the attracting molecular interaction potential wells we recently found by mathematical analysis performed on sptPALM data (Hoze et al., 2012).

Then, we investigated the temporal stability of nanodomains using time-lapse sptPALM. We performed $50 \mathrm{~s}$ periods of sptPALM acquisitions every 5 min over a time period of $45 \mathrm{~min}$. We could identify and track individual nanodomains in the spine for several tens of minutes (Fig. $6 A, B$ ). As quantified in Figure $5 D$, $>20 \%$ of the spines $(n=75)$ had a stable nanodomain during the entire acquisition time (45 min). Approximately $40 \%$ of the spines contained nanodomains, which could not be detected for longer than $5 \mathrm{~min}$ of observation, indicating that a fraction of the nanodomains in the spine are either dynamic or transient, whereas others have a very stable localization and lifetime. Nanodomains in the remaining $60 \%$ of the spines were stable for $>5$ min inside the synapses. More than $50 \%$ of spines had multiple nanodomains, bearing combinations of stable and nonstable nanodomains in the same synapse (Fig. 5D). Kymographs performed on highly stable nanodomains illustrate that nanodomains are visited by many molecules (Fig. 6A, $B$ ).

Finally, we analyzed the differences of mobility between in and out nanodomains, from single-molecule trajectories of Eos::GluA1 molecules obtained by sptPALM. Most of the immobilized molecules resided within the nanodomains, whereas molecules that were either outside or moving in and out of the nanodomains were either diffusive or weakly confined (Fig. 6C). We categorized the trajectories into three families, based on the nanoscale spatial heterogeneity as follows: (1) exclusively inside the nanodomain (In), (2) exclusively outside nanodomain (Out), and (3) those who visited the nanodomains for a minimum of $10 \%$ and maximum of $80 \%$ of the duration of their observed trajectories (In/Out). The cumulative distribution of the instantaneous diffusion of the trajectories displayed a shift toward lower mobility within, and higher mobility outside the nanodomains (Fig. 6D). The instantaneous diffusion distributions of In/Out molecules indicated an intermediate shift of mobility compared with In and Out ones (Fig. 6D). We also plotted the average of mean square diffusion curves for each of the three families of receptors (In, Out, and In/Out). The mean square diffusion of the molecules within nanodomains (In) displayed a strong confinement indicating immobilization (Fig. 6E). Receptors outside nanodomains (Out) exhibited unhindered Brownian diffusion. The mean square diffusion of receptors temporarily associated with nanodomains (In/Out) displayed weak confinement, indicating the transient changes in the mobility between strong confinement and unhindered diffusion. These data con- firm that nanodomains are indeed zones of strong confinement where AMPA receptors are transiently trapped with the potential for receptor to exchange and diffuse freely into the membrane outside the nanodomain.

\section{Nanoscale co-organization of PSD95 and AMPARs}

In the previous sections, the synaptic localization of nanodomains by super-resolution light microscopy was achieved with the epifluorescence image of Homer1c::Cerulean. Even though Homer1c is a postsynaptic protein, recent results indicate that it might not be closely associated with the PSD (Dani et al., 2010). To monitor the distribution of AMPAR nanodomains within the PSD, we used PSD95, a key organizer for postsynaptic scaffolding. PSD95 is also involved in the positioning and synaptic retention of glutamate receptors within the PSD and is localized closer to the postsynaptic membrane than Homer1c (Dani et al., 2010). We investigated the organization of PSD95 in spines by performing sptPALM on neurons expressing PSD95 genetically fused with mEos2 (Eos::PSD95). Epifluorescence images showed that the majority of Eos::PSD95 was localized to spine heads (Fig. 7A; Epifluorescence). Corresponding images obtained using sptPALM displayed similarly strong Eos::PSD95 labeling in spine heads with $>80 \%$ of the molecules observed by sptPALM located in spines and sparse labeling outside (Fig. 7A; sptPALMIntensity). At the spine level, most of the Eos::PSD95 signal was concentrated into a single large zone or, more rarely, in multiple zones (Fig. 7B). The area of Eos::PSD95 clusters was computed using a combination of thresholding and morphometry analysis on sptPALM intensity images. The size of Eos::PSD95 clusters ( $n=317$ synapses) followed a Gaussian distribution centered on $0.0405 \pm 0.025 \mu \mathrm{m}^{2}$ (Fig. $7 C$ ), with the mean of the principal and auxiliary axis calculated to be $\sim 424.7 \pm 177.2 \mathrm{~nm}$ (Fig. $7 C$, inset) and $282.8 \pm 78.85 \mathrm{~nm}$, respectively. These measurements are consistent with previous reports on size of the PSD (Petersen et al., 2003). Thus, we concluded that Eos::PSD95 is a reliable marker of the PSD.

A line-scan through the Eos::PSD95-labeled PSDs indicated that within PSDs, PSD95 is heterogeneously distributed (data not shown, but see Fig. 7J for similar results obtained with dSTORM on endogenous PSD95). At the subdiffraction limit scale, PSD95 intensity distribution fluctuated from a basal level to several higher intensity peaks, suggesting organization into subdomains. These subdomains were measured by anisotropic bidimensional Gaussian fitting (Fig. 7D) as described in Materials and Methods. The principal and auxiliary axes of PSD95 subdomains $(n=465)$ were, respectively, $158.2 \pm 100 \mathrm{~nm}$ (Fig. 7E) and $102.2 \pm 39 \mathrm{~nm}$. For comparison, the distribution of AMPAR nanodomain sizes, which were smaller, is also plotted in Figure $7 E$ (solid line). Finally, the number of PSD95 subdomains was counted for each of the Eos::PSD95-labeled PSDs, revealing that $>90 \%$ of PSDs contained one or more subdomains (Fig. $7 E$, inset). We also took advantage of sptPALM experiments to quantify the mobility of Eos::PSD95. As shown previously (Sharma et al., 2006), PSD95 showed low mobility in synapses and the distribution of PSD95 mobility within synapses was lower than the synaptic mobility of AMPARs (Fig. 7F).

To compare the distribution of PSD95 with respect to that of AMPARs at the PSD, we performed two-color dSTORM imaging (Heilemann et al., 2005; van de Linde et al., 2011) on neurons stained with antibodies for endogenous surface GluA1 coupled to Alexa-647 and for endogenous PSD95 to Rhodamine Red. As with the other super-resolution light imaging techniques, AMPAR nanodomains of comparable size and frequency were 


\section{A}
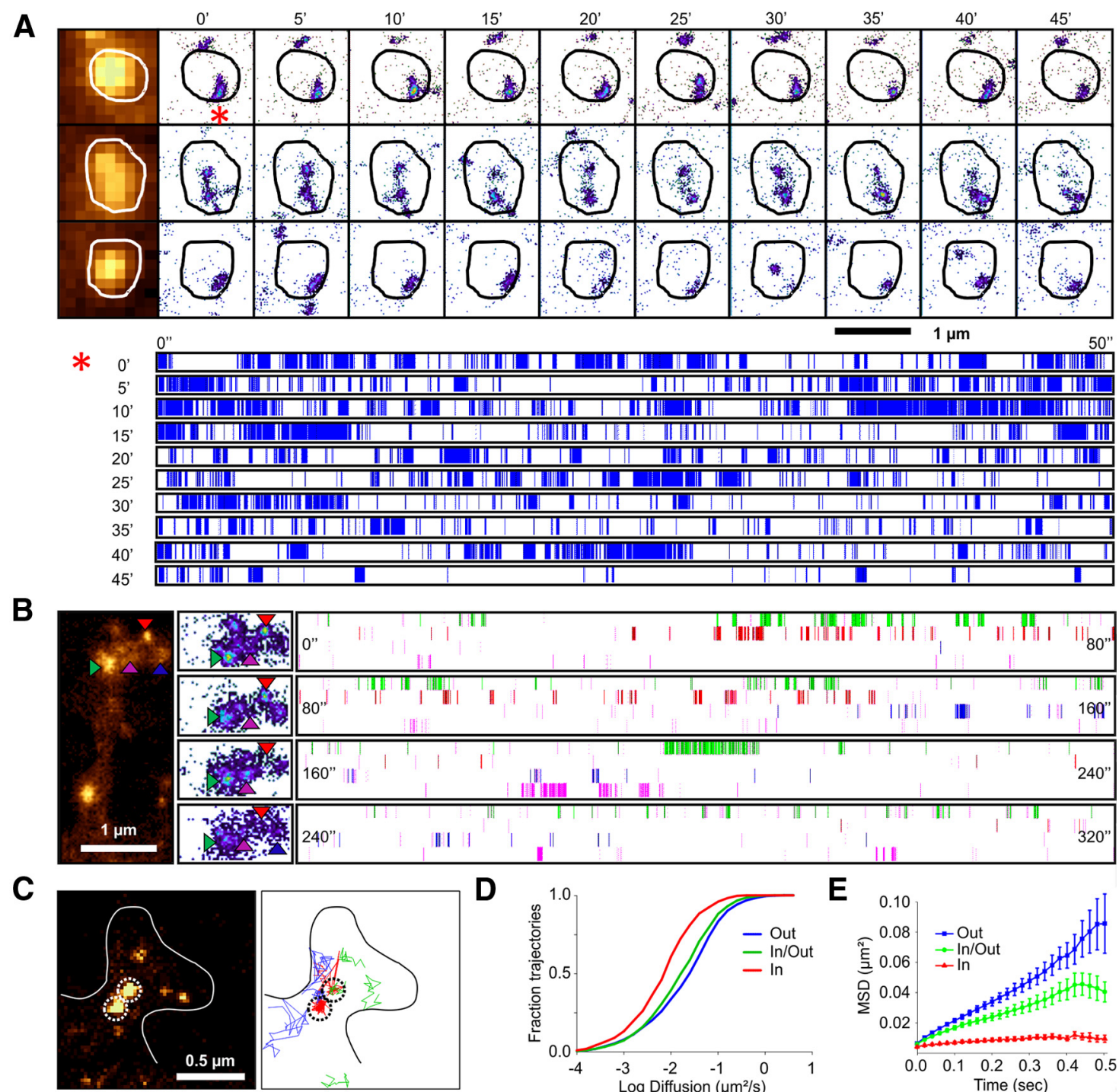

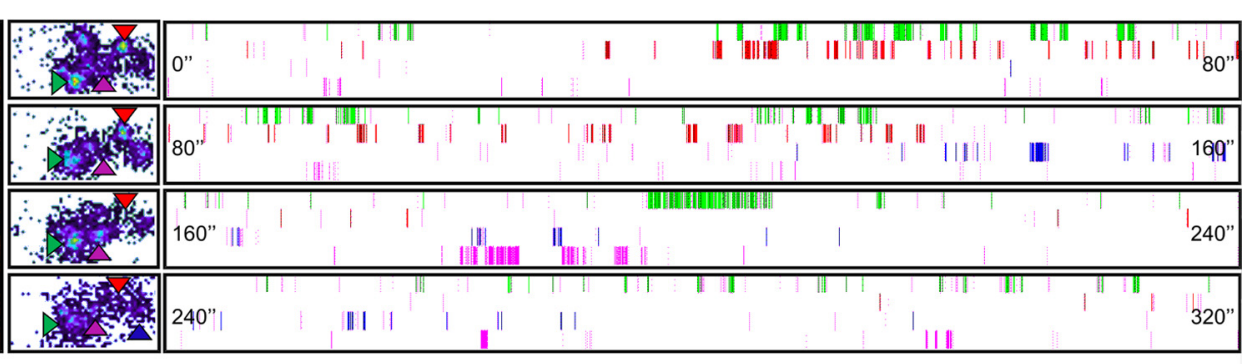
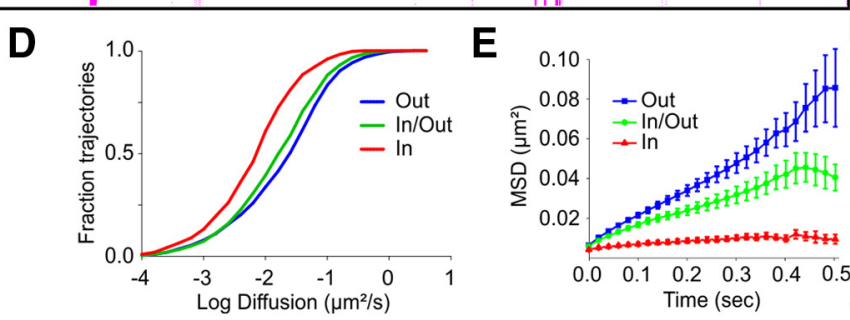

Figure 6. Time-lapse sptPALM recordings reveal stability of nanodomains. $A$, Examples of time-lapse sptPALM on three different synapses and the corresponding kymographs showing the presence of single molecules inside one nanodomain over time. Left side column, Epifluorescence images of the synapses labeled by Homer1c::Cerulean. Pseudo-color galleries represent the Eos::GluA1 super-resolution sptPALM intensity images acquired during 50 s every $5 \mathrm{~min}$. It shows that some nanodomains remain stable for the time of observation (first row), whereas the second and third rows display a more dynamic behavior of nanodomains or spine, including appearance and disappearance events. The kymograph displays the detection of fluorescent single molecules over time inside a nanodomain identified by the red star. Several possible patterns of single molecule fluctuations are visible along the time course, confirming that each nanodomain is a result of immobilization of several Eos-tagged GluA1 subunits. B, Kymograph of multiple nanodomains on a single spine extracted from a sptPALM experiment. Left side image, Super-resolution sptPALM intensity image of Eos::GluA1. The second column represents the super-resolution sptPALM intensity image sequence of the corresponding spine head every $80 \mathrm{~s}$. The arrows point to different nanodomains. Right column, Kymographs computed for each nanodomain represented by their respective color. The patterns of fluctuations denote that, whereas some domains are highly stable over time, other domains appear and disappear in the time range of minutes. C, Left panel, sptPALM intensity image of a spine expressing EOS::GluA1. Nanodomains are identified by a dashed circle. Right panel, Projection of a subset of single-molecule trajectories observed in the corresponding spine. Red represents strongly confined trajectories; blue and green represent diffusive and weakly confined trajectories, respectively. D, Cumulative distribution of instantaneous diffusion coefficients of the trajectories observed exclusively inside nanodomains (In), exclusively outside nanodomains (Out), or exchanging between inside and outside nanodomains (In/Out). $\boldsymbol{E}$, Average mean square diffusion plots for single-molecule trajectories observed exclusively inside nanodomains (In), exclusively outside nanodomains (Out), or exchanging between inside and outside nanodomains (In/Out).

detected by dSTORM (Fig. $7 H$; nanodomains size: $87 \pm 6 \mathrm{~nm}$ and $1.2 \pm 1.0$ nanodomains per spine, $n=87$ spines). Like Eos::PSD95, endogenous PSD95 exhibited a heterogeneous distribution inside the PSD with higher concentrations in subdomains (Fig. 7I,J). Although AMPARs were strictly localized to nanodomains and had low basal levels in the regions outside the PSD and outside nanodomains within the PSD, the basal level of PSD95 was higher between subdomains in the PSD than outside the PSD.

When viewed at the epifluorescence level, PSD95 and GluA1 displayed a high degree of overlap (Fig. $7 G$ ). However, dSTORM images revealed a varying degree of overlap between PSD95 and GluA1 (Fig. $7 \mathrm{H}, I$ ). A detailed analysis of a series of individual PSDs (Fig. $7 I, J$ ) indicated that PSD95 and AMPARs exhibited only partial colocalization. In many instances, the AMPAR nanodomains overlapped with the PSD95 subdomains, indicating an organizational relationship between the two proteins at the synapse (Fig. 7J). However, this perfect colocalization at the nanoscale is not systematic; AMPAR nanodomains and PSD95 subdomains were often tens of nanometers away from each other. This illustrates differences in the synaptic organization of AMPARs and PSD95. This result is at variance with a recent study that reported similar 
A

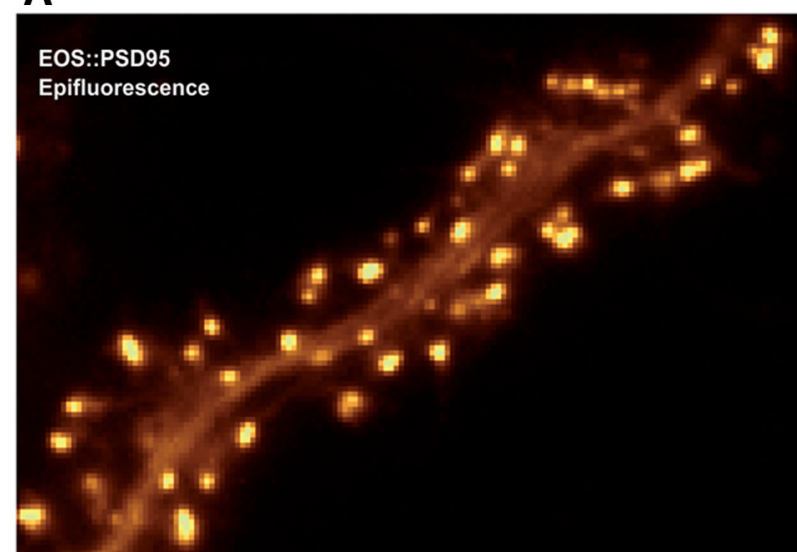

B

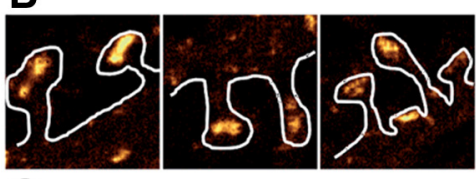

C

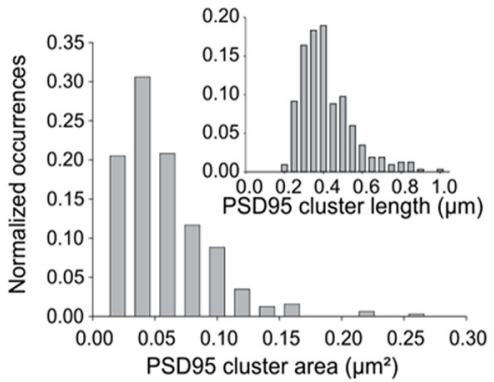

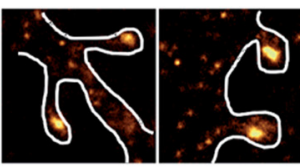

E

$0.35 \quad 0.6$

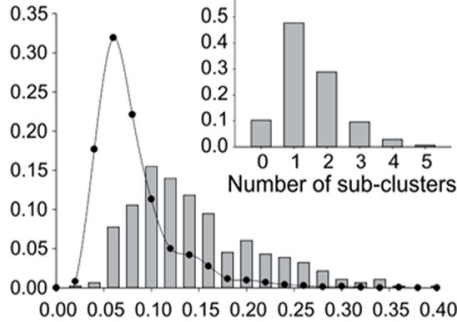

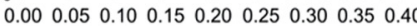
PSD95 sub-cluster length $(\mu \mathrm{m})$

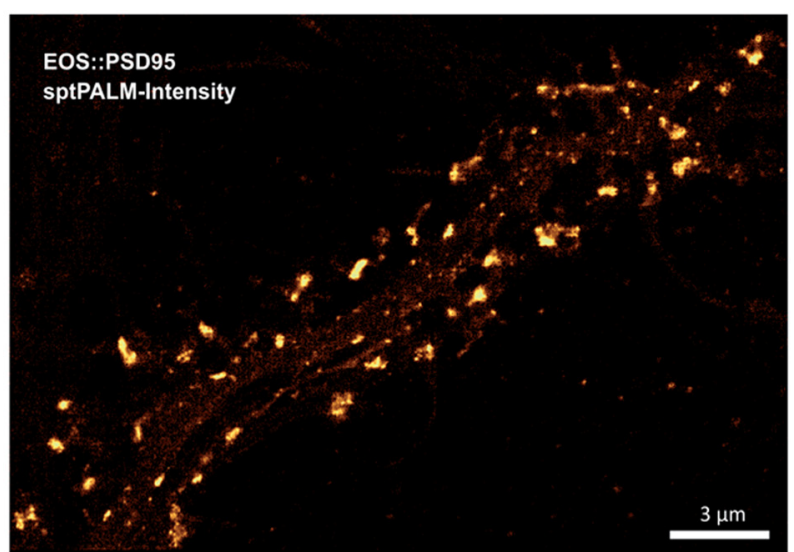

D

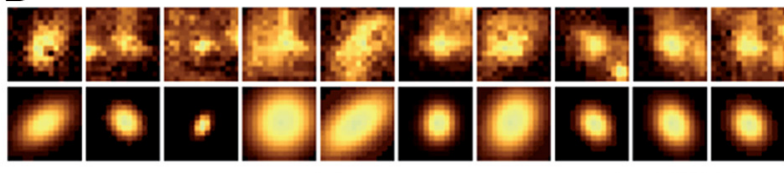

$\mathbf{F}$

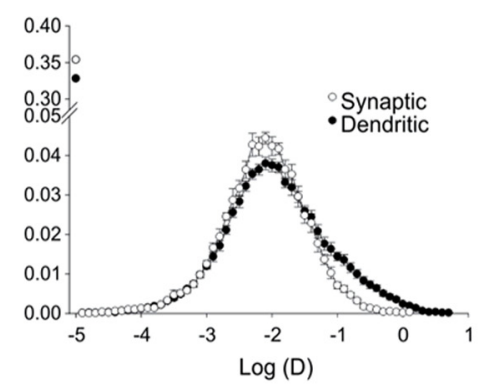

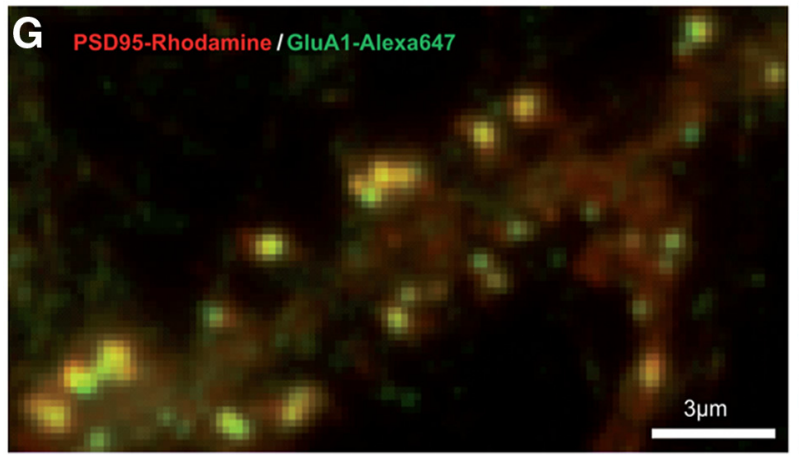
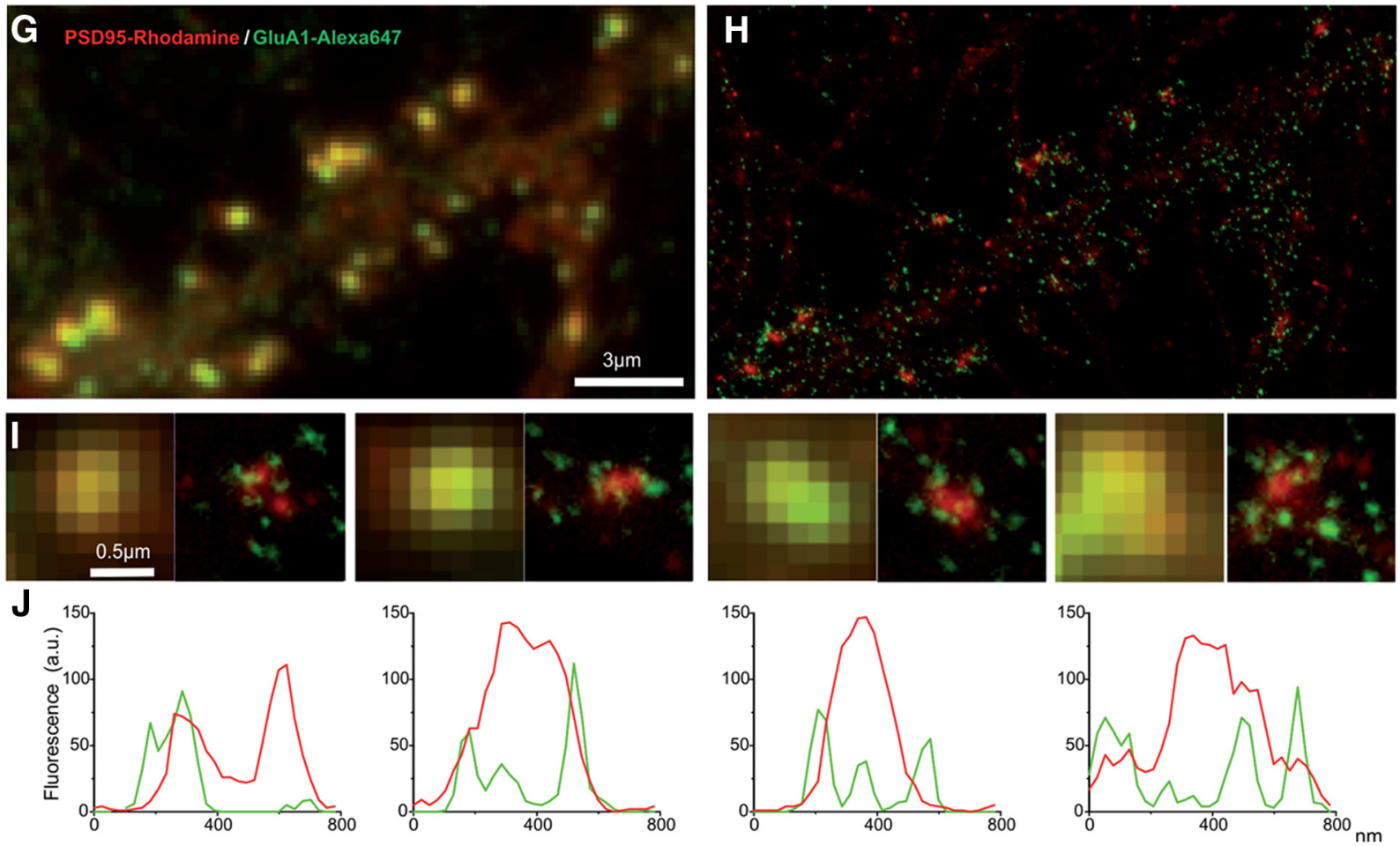

Figure 7. Subdiffraction quantification of PSD95 organization in spines and comparison with endogenous AMPARs. A, Comparison between epifluorescence and super-resolution intensity image of a neuron expressing Eos::PSD95. B, Gallery of super-resolution images of spines imaged by sptPALM (Eos::PSD95). C, Distribution of the area of the clusters of PSD95 from the spine with inset representing the distribution of the cluster lengths. $\boldsymbol{D}$, Examples of PSD95 subclusters acquired by sptPALM and the corresponding fit performed using $2 D$ anisotropic Gaussian functions. $E$, The principal axis (length) of PSD95 subclusters obtained by $2 \mathrm{D}$ anisotropic Gaussian fitting. The continuous line represents the corresponding distribution of GluA1 subunit (Figure legend continues.) 

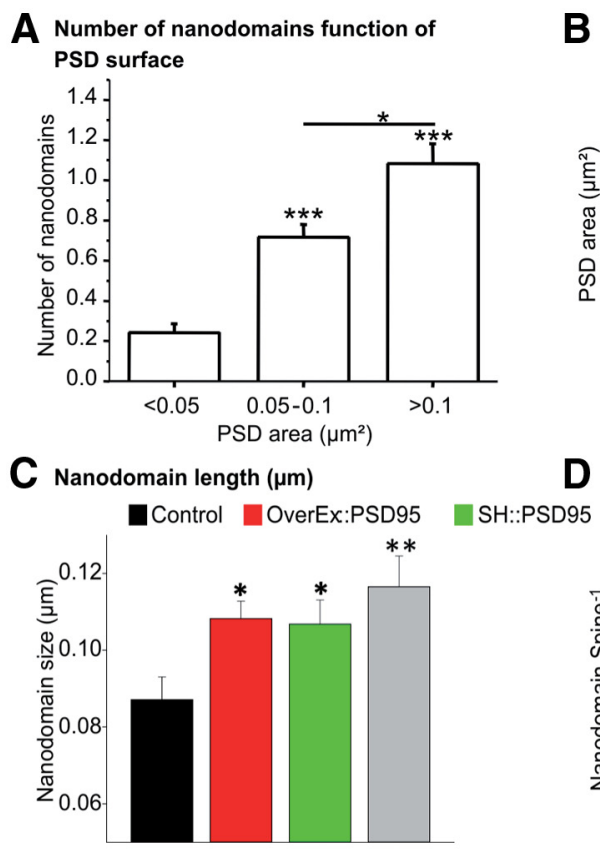

\section{E Synaptic endogenous GluA2}

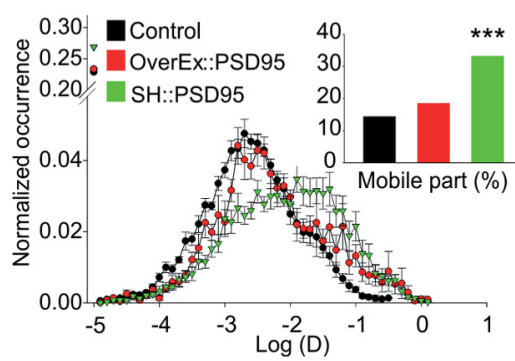

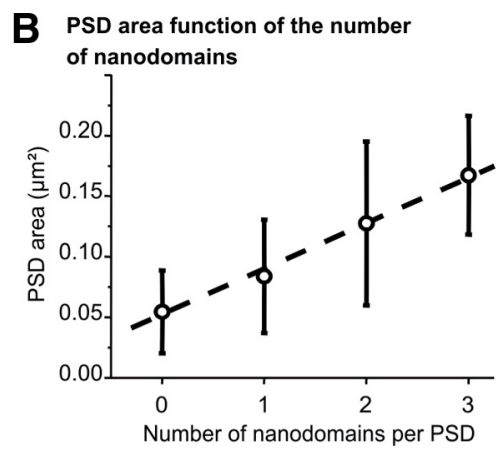

B PSD area function of the number

D Number of nanodomains per spine
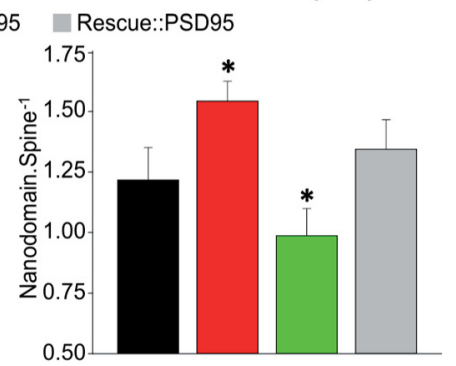

F Nanodomain size

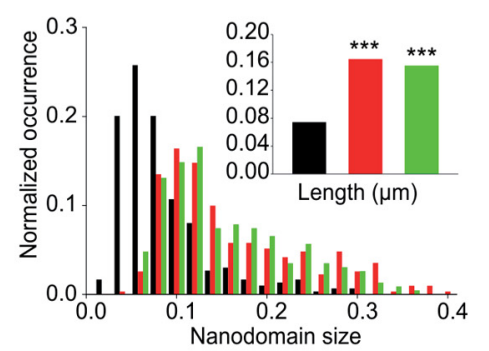

Figure 8. Correlation of PSD size with AMPAR nanodomains and effects of the modulation of endogenous PSD95 on nanodomain characteristics. $A$, Plot of the number of nanodomains per PSD for various ranges of sizes of the PSD measured from the PSD95 staining using dual-color dSTORM. Significant differences were observed between each dataset $(p<$ 0.05). $\boldsymbol{B}$, Plot of the number of nanodomains as a function of the PSD area. Interestingly, the data nicely fit with a linear model (dashed line). These data indicate that the PSD area is directly proportional to the number of nanodomains. $\boldsymbol{C}$, Comparison of nanodomain length between control and modulation of endogenous PSD95 levels. Black, red, green, and gray represent control, OverEx::PSD95, SH::PSD95, and Rescue::PSD95, respectively. $\boldsymbol{D}$, Variability of the number of nanodomains per spine for different experimental conditions. $E$, Distributions of synaptic diffusion coefficients of endogenous AMPAR measured by uPAINT, for different experimental conditions. Neurons were transfected with Homer-DsRed as a synaptic marker. Inset, Mobile fraction of receptors for each condition. $\boldsymbol{F}$, Distributions of nanodomain lengths measured by UPAINT on live neurons with different levels of PSD95 expression. Average values of nanodomain lengths are plotted in the inset. ${ }^{*} p<0.05 .{ }^{* *} p<0.01 .{ }^{* *} p<0.001$.

nanodomains than in our work but found a high degree of colocalization between AMPAR and PSD95 (Macgillavry et al., 2013). This may arise from the fact that this study used overexpressed PSD95 or AMPARs, manipulations that may alter the normal reciprocal distribution of both elements.

Next, we correlated the endogenous PSD area labeled by PSD95 with the number of AMPAR nanodomains from the two-color dSTORM super-resolution images. PSDs were grouped into three different size categories: $<0.05,0.05-0.1$, and $>0.1 \mu \mathrm{m}^{2}$. The number of nanodomains per group was $0.24 \pm 0.04,0.72 \pm$ 0.06 , and $1.08 \pm 0.78$, respectively. These data indicated that small PSDs were more likely to contain less or no nanodomains compared with larger ones (Fig. 8A). Interestingly, the number of nanodomains per PSD increased linearly with the PSD area (correlation coefficient $=0.997$, $\mathrm{SD}=0.107)$ (Fig. $8 B$ ). Finally, we measured the distance between centroids of nanodomains contained within the same spine or the same PSD. The average distance between the nanodomains contained within a spine was $512.88 \pm 26.5$ $\mathrm{nm}(n=91$ spines). In $<30 \%$ of the cases, the distance between nanodomains within a spine was $<250 \mathrm{~nm}$ (median 455 nm, IQR 309.11/732.7, $n=91$ ). When looking specifically at nanodomains within the same PSD95 cluster, the average distance between nanodomains was $317.30 \pm$ $168.34 \mathrm{~nm}$, and the average PSD diameter was $339.14 \pm 228.89$ (18 PSDs). In $\sim 50 \%$ of the cases, the distance between nanodomains in the same PSD was $<250 \mathrm{~nm}$ (median $247.6 \mathrm{~nm}$, IQR 191.1-439.7 nm), confirming that nanodomains are more concentrated in PSDs than overall in the spine. The average size of the PSDs obtained by endogenous PSD95 staining was smaller than that obtained by overexpression of mEos::PSD95. This is consistent with the observation that PSD95 overexpression can increase the size of the dendritic spines (El-Husseini et al., 2000).

\footnotetext{
(Figure legend continued.) nanodomains obtained by sptPALM. Inset, Distribution of PSD95 subclusters per cluster obtained by sptPALM. $F$, Distributions of the synaptic (white) versus the dendritic mobility (black) of PSD95 obtained by sptPALM. $\boldsymbol{G}, \boldsymbol{H}$, Comparison of colocalization between endogenous PSD95 (red) and endogenous surface GluA1 (green) between an epifluorescence image $(\boldsymbol{G})$ and the corresponding dSTORM super-resolution image $(\boldsymbol{H})$. $\boldsymbol{I}$, Gallery of comparison of individual synapses for colocalization between endogenous PSD95 (red) and endogenous surface GluA1 (green). For better visibility, GluA1 super-resolution images are depicted with a $\gamma$ of 2.J, Line-scans on the corresponding super-resolution images in the above gallery illustrating that PSD95 (red) is not uniformly distributed within the synapse but organized in subclusters, indicating heterogeneity in the distribution of endogenous PSD95 within PSD. In contrast to PSD95, GluA1 (green) shows a nanodomain distribution with a higher contrast between intensities in the nanodomain versus extra nanodomain regions. It can also be observed in the line-scan that in many cases nanodomains of GluA1 overlap with subclusters of the PSD95, whereas domains with no overlap are also visible.
}

\section{Modulation of endogenous PSD95 levels and its effect on AMPAR nanodomains}

PSD95 is one of the core components of the PSD and could thus play a role in organizing AMPAR nanodomains. To test this hypothesis, we modulated the level of PSD95 in neurons using shRNA to reduce PSD95 levels or overexpression of PSD95. An shRNA-resistant PSD95 rescue condition was included to verify the specificity of shRNA effects. These conditions will be referred to as SH::PSD95, OverEx::PSD95, and Rescue::PSD95. Immunofluorescence staining for PSD95 was used to measure variations in PSD95 expression level. In cells expressing SH::PSD95, we observed a $20-25 \%$ reduction in the fluorescence intensity of endogenous PSD95 compared with 
Table 1. Comparison of endogenous AMPAR nanodomain properties upon modulation of endogenous levels of PSD95 by dSTORM

\begin{tabular}{lclll}
\hline Sample & $\begin{array}{l}\text { Nanodomain } \\
\text { size }(\mathrm{nm})\end{array}$ & $\begin{array}{l}\text { No. of nanodomains/ } \\
\text { spine }\end{array}$ & $\begin{array}{l}\text { No. of } \\
\text { cells }\end{array}$ & $\begin{array}{l}\text { No. of } \\
\text { cultures }\end{array}$ \\
\hline Control/no transfection & $87 \pm 6$ & $1.21 \pm 0.13$ & 16 & 4 \\
Homer 1c::GFP & $92 \pm 3$ & $1.18 \pm 0.07$ & 9 & 2 \\
SH::Scramble::GFP & $89 \pm 7$ & $1.07 \pm 0.14$ & 8 & 2 \\
SH::Scramble::Homer1c & $89 \pm 7$ & $1.07 \pm 0.13$ & 5 & 2 \\
ShPSD95:::GFP & $107 \pm 6$ & $0.98 \pm 0.11$ & 10 & 3 \\
Rescue:::PSD95::GFP & $116 \pm 8$ & $1.34 \pm 0.12$ & 8 & 2 \\
Overexpression PSD95::GFP & $108 \pm 5$ & $1.54 \pm 0.08$ & 7 & 2 \\
\hline
\end{tabular}

nontransfected cells. In neurons expressing Rescue::PSD95 and OverEx::PSD95, we observed a 6- to 7-fold and 15- to 17-fold, respectively, increase in PSD95 levels compared with nontransfected cells. These results were consistent with previous reports using these DNA constructs (Schluter et al., 2006; Mondin et al., 2011).

To quantify the effect of PSD95 knockdown or overexpression on AMPAR nanodomains, we performed dSTORM imaging of immunostained surface GluA2 (endogenous) in neurons expressing the various constructs. The average length of the nanodomains significantly increased from control neurons compared with neurons expressing SH::PSD95, OverEx::PSD95, and Rescue::PSD95 (Table 1; Fig. 8C; Mann-Whitney test, $p=$ $0.017, p=0.039$, and $p=0.015$, respectively). Although the Rescue::PSD95 resulted in larger nanodomains $(116 \pm 8 \mathrm{~nm})$, this increase was found to be not significant compared with SH::PSD95 and Overex::PSD95. The number of nanodomains per spine was also significantly affected by the modulation of PSD95 levels (Table 1; Fig. 8D). Nanodomain number decreased in cells expressing SH::PSD95 compared with control ones (Table 1 ; Fig. $8 D$; control number of nanodomain/spine $1.21 \pm 0.13$, SH-PSD95 $0.98 \pm 0.11, p=0.046)$. In contrast, the number of nanodomains per spine increased dramatically in cells expressing OverEx::PSD95 (Table 1). Although expression of the Rescue::PSD95 displayed an increase in the nanodomain density of $1.34 \pm 0.12$ compared with the control, this increase was not found to be significant (Table 1).

The coexpression of a generic scrambled shRNA against a noneffective mammalian protein with GFP (SH::Scramble::GFP) or with another synaptic protein Homer 1c::GFP (SH::Scramble:: Homer1c) displayed no significant changes in the nanodomain characteristics compared with the control untransfected cells or cells expressing Homer1c::GFP (Table 1). No significant difference was detected either in the nanodomain size or number between OverEx::PSD95 and Rescue::PSD95, indicating that the Rescue::PSD95 mimics mild overexpression of PSD95. Consequently, the dynamic experiments on live cells and further quantifications were performed using control, SH::PSD95, and OverEx::PSD95 unless otherwise stated.

We also estimated the surface covered by nanodomains in spines. In control conditions, nanodomains covered $28 \pm 2.08 \%$ of the spine surface and concentrated $65.7 \pm 3.11 \%$ of AMPAR signal ( $n=8$ neurons). For the whole neurons, the nanodomains accounted for $43 \pm 5.14 \%$ of the total AMPAR signal but represented only $2.09 \%$ of the total observed neuronal surface. Interestingly, on cells overexpressing PSD95, AMPARs were more clustered as nanodomain content increased to $55.1 \pm 4.62 \%$ of the total AMPAR signal in only $1.27 \%$ of the neuronal surface. $\mathrm{SH}:$ :PSD95 expression induced a reduction in clustering as nanodomain content decreased to $36 \pm 3.52 \%$ of AMPARs signal, present on $0.98 \%$ of the total neuronal surface. This indicated
Table 2. Comparison of endogenous AMPAR nanodomain properties upon the modulation of stargazin-PSD95 interaction by UPAINT

\begin{tabular}{lcc}
\hline Sample & Density $(\text { nanodomains/ } \mu \mathrm{m})^{2}$ & Nanodomain size $(\mathrm{nm})$ \\
\hline Control/Homer::GFP & $0.13 \pm 0.03$ & $74 \pm 1$ \\
PSD95H225V & $0.08 \pm 0.03$ & $112 \pm 5$ \\
STG T231F & $0.007 \pm 0.002$ & $135 \pm 12$ \\
PSD95H225V + STG T231F & $0.03 \pm 0.01$ & $170 \pm 14$ \\
\hline
\end{tabular}

that overexpression of PSD95 concentrated AMPARs in nanodomains, whereas reduction of PSD95 reduced the number of AMPARs on the neuronal membrane as well as in nanodomains.

Relationship between PSD95 expression level and association with stargazin on the dynamic organization of AMPARs at the synapse

The intracellular scaffold protein PSD95 has been suggested to play a major role in stabilizing AMPARs at synaptic sites through binding to their auxiliary TARP (Schnell et al., 2002; Bats et al., 2007; Opazo et al., 2012). To investigate the role of PSD95 in the dynamic organization of nanodomains, we quantified AMPAR dynamics in neurons with uPAINT while modulating PSD95 levels. We computed the distribution of GluA2 mobility globally (on the whole neuron) and selectively inside synapses (Fig. 8E) and compared it with GluA2 mobility in control neurons expressing Homer-DsRed or neurons expressing OverEx::PSD95 and SH::PSD95. When OverEx::PSD95 was expressed, the global mobility of AMPARs was slower than in control neurons. No significant difference in AMPAR mobility was observed at synapses, probably because of the already saturated concentration of PSD95 (global median values Homer-DsRed, $n=18$ cells, 0.0012 IQR 0.00001-0.007, synapses 0.0008 IQR 0.00001-0.0048; Homer-DsRed + OverEx::PSD95, $n=14$ cells, 0.0009 IQR 0.00001-0.0052, synapses 0.00076 IQR 0.00001-0.005). Expression of SH::PSD95 induced the opposite effect. Both the global and synaptic mobility of AMPARs was significantly increased (Homer-DsRed + SH::PSD95, $n=15$ cells, 0.0034 IQR $0.00006-$ 0.021, synapses 0.0023 IQR 0.00001-0.011).

We next analyzed variations in AMPAR nanodomain size in spines as a function of PSD95 expression level using uPAINT (Fig. $8 F$ ). The average length of the nanodomain principal axis increased from $74 \pm 1 \mathrm{~nm}$ for control spines to $164 \pm 5 \mathrm{~nm}$ for OverEx::PSD95 $(n=311)$ and $155 \pm 4 \mathrm{~nm}$ for SH::PSD95 $(n=$ 229) (Fig. $8 F$, inset). These experiments confirm the quantifications obtained from dSTORM data and show that both increase and decrease in PSD95 expression levels trigger a significant increase in AMPAR nanodomain size.

We demonstrated previously that overexpression of a mutant form of stargazin (StgT231F) (Schnell et al., 2002; Bats et al., 2007), which prevents C-terminal association of stargazin to PSD95, increases the mobility of AMPARs globally and at synapses (0.04 IQR 0.0036-0.15 synapses 0.035 IQR 0.006-0.12), demonstrating that the interaction between stargazin and PSD95 is critical for AMPAR immobilization. Here, we examined the effect of StgT231F expression on the density and size of nanodomains (Table 2). StgT231F induced a major reduction in nanodomain density compared with the control (control $0.13 \pm 0.03$ nanodom $\cdot \mu \mathrm{m}^{-2}$ and StgT231F $\left.0.007 \pm 0.002, p<0.001\right)$. On the contrary, coexpression of StgT231F with a rescue variant of PSD95 (PSD95H225V), which can bind StgT231F (Schnell et al., 2002), partially rescued global nanodomain density and AMPAR immobilization (0.03 IQR 0.0032-0.14 synapses 0.028 IQR $0.0039-0.11)$. The coexpression of StgT231F and PSD95H225V 
A

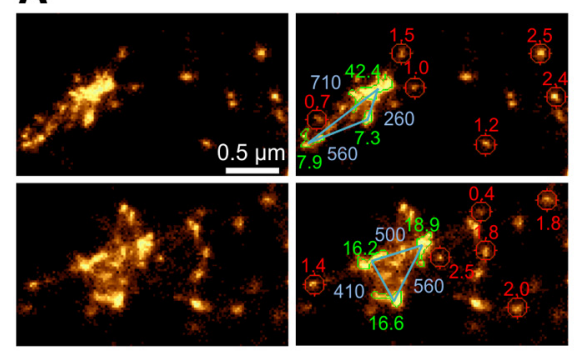

B

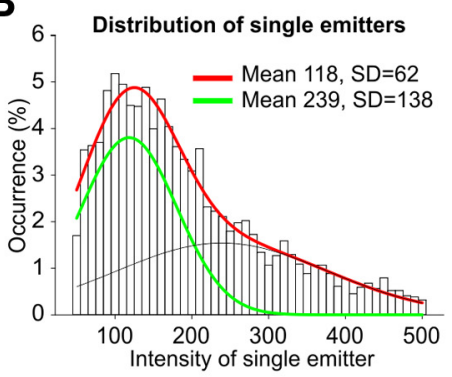

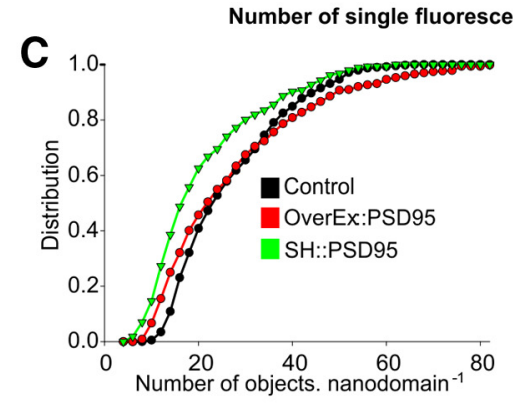

ers per nanodomain
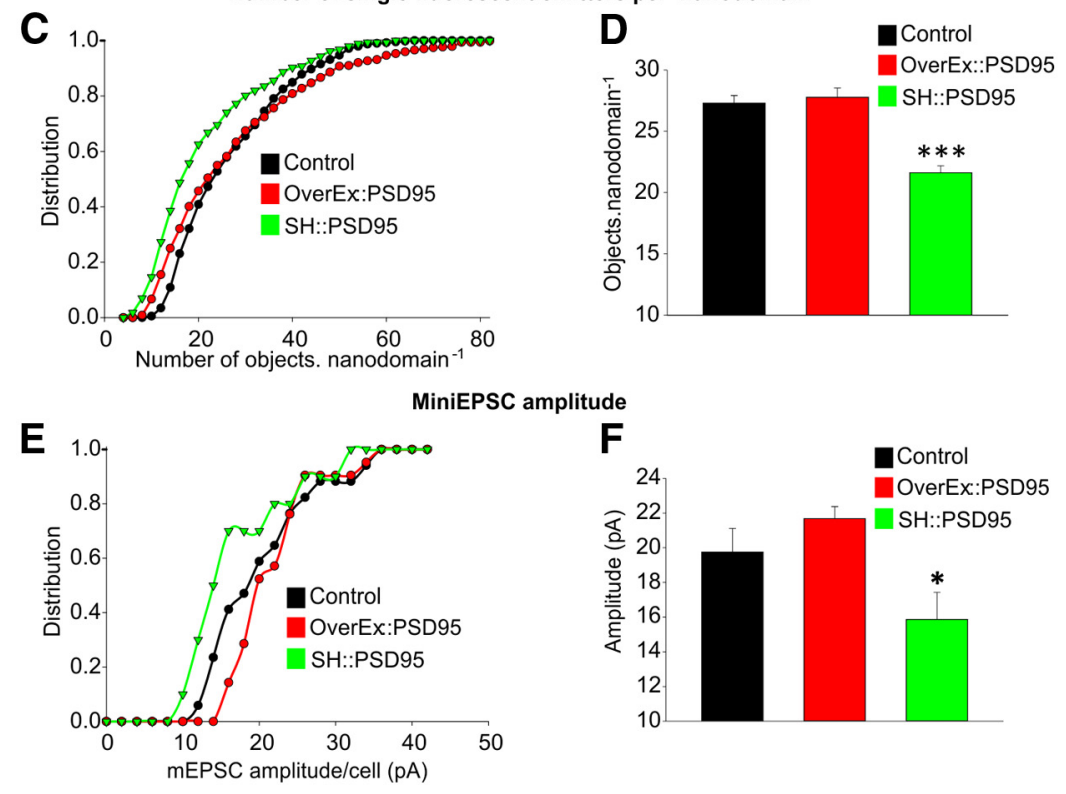

Figure 9. Estimation of number of AMPAR per nanodomain and correlation with mEPSC.A, Localization of AMPAR single emitters and nanodomains and estimate of their AMPAR content. Left column shows two spines labeled with surface endogenous GluA2 reconstructed from ASTORM. Right column displays the estimate of AMPAR content for some single isolated emitters (red) and nanodomains (green), together with distances between nanodomain (in $\mathrm{nm}$, blue). In these examples, nanodomain contentvaries between 7.3 and 42.2AMPARs and the distances between nanodomains range from 260 to $710 \mathrm{~nm}$. B. Intensity distribution of single fluorescent emitters outside nanodomains, in the dendritic shaft. The distribution fits with a sum of two Gaussian distributions, the second one being centered on twice the center of the first one. C, Histograms of AMPAR content estimate inside nanodomains for control (black) and SH::PSD95 (green) conditions. The estimated number of molecules per nanodomain is reduced in the case of SH::PSD95 compared with the control condition. D, Variation of AMPAR density in nanodomains for various levels of endogenous PSD95 expression. ${ }^{* * *} p<0.001$. E, mEPSC distribution obtained by patch-clamp recordings on neurons expressing the same constructs as in Figure $8 C, D$. $F$, Average values of the mEPSCs are plotted for various modulations of endogenous PSD95 levels. ${ }^{*} p<0.05$.

also resulted in a large increase in the global density of nanodomains compared with the StgT231F expression alone (coexpression $0.03 \pm 0.01$ compared with Stg T231F $0.007 \pm 0.002$, $p=0.042$ ). The expression of PSD95H225V alone did not significantly affect the mobility of AMPA receptors globally or at synapses (0.007 IQR 0.00003-0.072 synapses 0.00358 IQR 0.000020.032 ). It also only had a minor effect on the global nanodomain density of AMPARs (Table 2). This is expected as only one of the PDZ domains in PSD95 is mutated while the other two PDZ domains can still bind to endogenous stargazin. On the contrary, StgT231F cannot bind to any of the PSD95 family PDZ domains, explaining its drastic effect on AMPAR organization.

Expression of StgT231F alone, PSD95H22V alone, or coexpression of StgT231F and PSD95H225V also increased the size of nanodomains (Table 2; $p<0.001$ for the three conditions compared with the control), similarly to the effect of modulating endogenous PSD95 levels, confirming that the stargazin-PSD95 interaction is vital for the formation of nanodomains as well as AMPAR immobilization.

Correlating AMPAR organization and synaptic transmission

Next, we sought to estimate the number of AMPARs per nanodomains from dSTORM super-resolution images obtained from surface GluA2 staining. As a reference for the signal provided by an individual labeled AMPAR, we measured the distribution and median of the fluorescence intensity arising from isolated single emitting species in the shaft membrane, as detailed in Materials and Methods (Fig. 9A, red circles). The signal variability includes fluctuations that depend on the antibody labeling efficacy and fluorophore blinking properties. The intensity distribution of these single emitters fit perfectly with a bimodal Gaussian distribution (Fig. 9B) with the second peak mean intensity located at twice the first peak mean intensity $(239.0 \pm 138.1$ and $118.4 \pm 62.11$, respectively, 9 cells, 4405 isolated AMPARs). This is coherent with the hypothesis that the signal from the isolated single emitting species arises from individual AMPARs that usually contain two GluA2 subunits and in which either one or both the GluA2 subunits could be labeled. Interestingly, the cell-tocell fluctuations of the median intensity was $<10 \%$. To estimate the AMPAR content per nanodomain, we used the median intensity value of this single emitter distribution for each cell, as a putative reference for single receptor fluorescence intensity. Figure $9 A$ illustrates various single receptors (red circles) and nanodomains (green regions) with their respective number of AMPAR estimation. We extracted the distribution of AMPARs per nanodomain for the control and the SH::PSD95 conditions (Fig. 9C). We observed a decrease of the number of AMPARs per nanodomain for the SH::PSD95 $(21.6 \pm 0.58, n=12$ cells) compared with the control conditions $(27.3 \pm 0.61, n=9$ cells), corresponding to a reduction of $21 \%$ (Fig. 9D). Interestingly, no significant difference was observed in the number of AMPARs per nanodomain between control and OverEx::PSD95 (27.76 $\pm 0.76, n=14$ cells) (Fig. 9D).

To correlate these fluctuations in nanodomain AMPAR content to synaptic responses, we measured the mEPSC amplitudes from neurons in similar conditions, and compared measurements in control, upregulated, or downregulated PSD95 expression level. For correlation purposes, we recorded cells from the same primary hippocampal cultures that were used for uPAINT experiments to quantify AMPAR dynamics and nanodomain size (Fig. $8 C, D$ ). The mEPSC amplitude distribution displayed a shift toward higher values when PSD95 was overexpressed compared with the control condition (Fig. 9E). mEPSC amplitude average values (Fig. 9F) revealed that OverEx::PSD95 is not significantly different from control (control, $n=17$ cells, $19.7 \pm 1.3 \mathrm{pA}$; OverEx::PSD95, $n=21$ cells, $21.7 \pm 0.7 \mathrm{pA}$ ). In contrast, PSD95 knock-down induced a $20 \%$ decrease in mEPSC amplitude 
(SH::PSD95, $n=14$ cells, $15.8 \pm 1.5 \mathrm{pA})$. Together, these experiments demonstrate a positive correlation between nanodomain AMPAR content and mEPSC responses.

\section{Modeling the impact of AMPAR organization on synaptic transmission}

To estimate the potential impact of AMPAR nanoscale organization on synaptic transmission, we performed Monte Carlo simulations as in Heine et al. (2008), but using various AMPAR organizations (Fig. 10). We used previously established parameters of glutamate release in the synaptic cleft, AMPAR activation, and desensitization kinetics schemes (Fig. 10A) (Jonas et al., 1993; Franks et al., 2002; Lisman et al., 2007; Heine et al., 2008). The model perfectly mimicked the kinetics and amplitude of experimentally recorded mEPSCs (Fig. $10 C)$. We then systematically varied the localization (Fig. $10 D-F$ ) and density (Fig. 10G-I) of an AMPAR nanodomain containing 25 receptors and also analyzed the crosstalk between multiple nanodomains located at varying distances (Fig. $10 \mathrm{~J}, \mathrm{~K})$. All parameters strongly impacted the amplitude of mEPSCs. However, our simulations indicate that there is a degree of tolerance on the location of the glutamate release site with respect to the AMPAR nanodomain because the amplitude of the mEPSC only starts to decrease when glutamate is released $>100 \mathrm{~nm}$ away from the nanodomain center (Fig. $10 D-F)$. In contrast, the mEPSC amplitude was very sensitive to AMPAR density because it started to decrease as soon as it was lower than the maximal packaging we used of one receptor every $20 \mathrm{~nm}$ (Fig. 10G-I).

We then modeled the presence of two similar nanodomains in the synapse. First, glutamate was released on top of one cluster and the second one positioned at varying distances. The second cluster had no more impact on the AMPAR response when it was distant of $>300 \mathrm{~nm}$ (Fig. $10 \mathrm{J1}$,2), which is about the mean value that we measured for intercluster distances. Second, we fixed the intercluster distance at $300 \mathrm{~nm}$ and varied the location of glutamate release. Interestingly, the AMPAR response was markedly increased when glutamate was released in between the two clusters (maximum 19.2 \pm 3.6 activated AMPARs) compared with the condition with a single cluster in the synapse (maximum $16.2 \pm 3.3$ activated AMPARs, $p<0.002$ ) (Fig. 10K1,2).

Together, our experimental data and these simulations indicate that AMPAR nanoscale organization as well as location of the glutamate release strongly impact mEPSC amplitude.

\section{Discussion}

Using four independent super-resolution light imaging methods on both genetically tagged and endogenous receptors, as well as pre-embedding EM, we demonstrate that, in live and fixed hippocampal neurons, AMPARs are organized inside most synapses into a few highly concentrated domains of $\sim 70 \mathrm{~nm}$, and not homogeneously distributed as previously described. AMPARs are stabilized in nanodomains and freely randomly diffuse between them. Nanodomains are dynamic in their composition and overall structure. AMPARs can enter and exit nanodomains within seconds by lateral diffusion. Inside synapses, nanodomains can have two different behaviors: they can be stable for up to an hour or change shape and move and/or disappear within minutes, albeit at rather slow rates. Nanodomain estimated size is robust as measures of AMPAR distribution by sptPALM and uPAINT using both localization maps and confinement of receptor movements from MSD curves yielded comparable values. This was confirmed on fixed cells using STED, dSTORM, and EM. The observation of similar clusters in fixed and living cells reinforces that they are functional receptor domains. Live-cell single molecule imaging demonstrates that nanodomains are dynamic as AMPARs could enter and exit them frequently. Previous low-density single particle tracking data showed the alternating trapping and free diffusion of AMPARs in the PSD (Groc et al., 2004), as well as confinement in subspine compartments (Ehlers et al., 2007). However, these data could not provide enough spatial information to map nanodomains inside synapses. A recent super-resolution light microscopy study using immunocytochemistry and STORM on fixed samples (Dani et al., 2010) has highlighted the molecular organization of many postsynaptic molecules, including AMPARs. Even though an inhomogeneous AMPAR distribution was observed, with clusters apparent on the micrographs, the heterogeneity of receptor staining within the synapses appeared lower compared with our study and the existence of nanodomains was not reported.

Studies of AMPAR distribution at the nanometer level using EM and either postembedding immunogold labeling (Kharazia et al., 1996; Petralia et al., 1997; Nusser et al., 1998; Takumi et al., 1999a) or pre-embedding labeling (Tao-Cheng et al., 2011) or SDS-digested freeze fracture replica labeling (Masugi-Tokita et al., 2007; Tarusawa et al., 2009; Budisantoso et al., 2012; Fukazawa and Shigemoto, 2012) have suggested a nonhomogeneous distribution of receptors within the PSD. SDS-digested freeze fracture replica labeling revealed a microcluster intrasynaptic arrangement of AMPARs in corticogeniculate synapses and a more diffuse arrangement in retinogeniculate synapses (Tarusawa et al., 2009). Similarly, AMPARs are organized in intrasynaptic microclusters in parallel fiber-Purkinje cell synapses, whereas they are homogeneously distributed in climbing fiber-Purkinje cell synapses (Masugi-Tokita et al., 2007). AMPAR density in the clusters reported in these preparations ranged from $\sim 500$ to 2000 AMPAR $/ \mu \mathrm{m}^{2}$. We found that AMPAR density inside nanodomains reached $\sim 2500 \mathrm{AMPAR} / \mu \mathrm{m}^{2}$, which is comparable. The number of nanodomains detected per synapse was slightly different in between the different techniques, although in the same range, with more domains detected by EM compared with dSTORM, sptPALM, and uPAINT. This can be explained by the more efficient detection efficacy reached by EM.

A novel approach to immunogold labeling in which neurons were live-labeled with a highly avid GluA2 primary antibody, followed by pre-embedding immunogold secondary application and silver intensification, resulted in high-density labeling of surface GluA2 and the presence of AMPAR nanodomains. The underestimation of the degree of AMPAR clustering in earlier EM studies (Kharazia et al., 1996; Petralia et al., 1997; Nusser et al., 1998; Takumi et al., 1999a) could arise from undersampling of the epitopes with postembedding methods and use of different primary antibodies. Importantly, our super-resolution experiments in live cells indicate that the AMPAR organization into nanodomains does not result from a fixation artifact.

The stabilization of AMPARs at postsynaptic sites is regulated by the interaction of AMPAR subunits and auxiliary proteins, such as TARPs, with intracellular and extracellular interactors (Nicoll et al., 2006; Bats et al., 2007; Frischknecht et al., 2009). AMPAR nanodomains are relatively stable, as they can last for at least an hour, and most likely originate from interactions of AMPAR complex components with intra- 
A

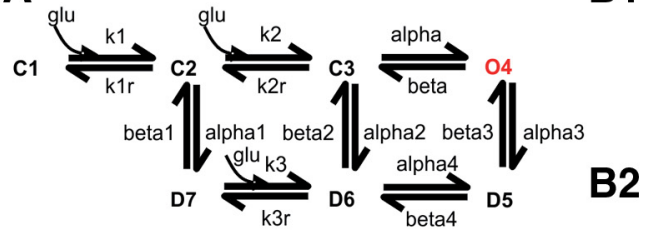

B1

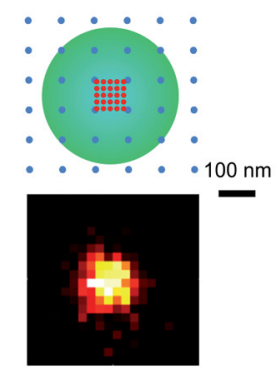

D

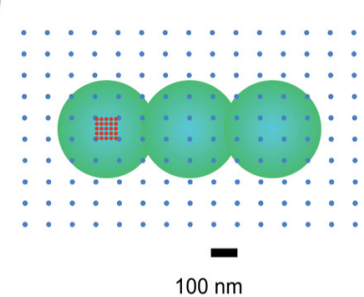

G

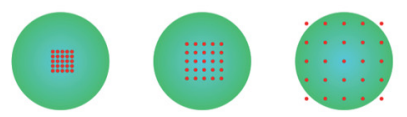

J1

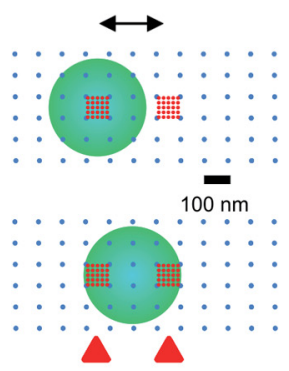

E

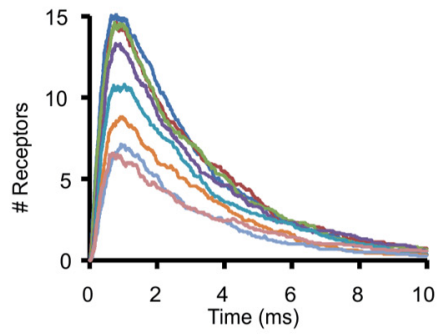

H

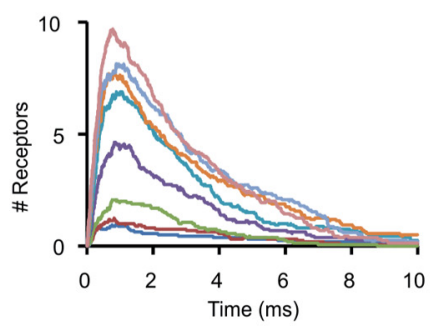

J2

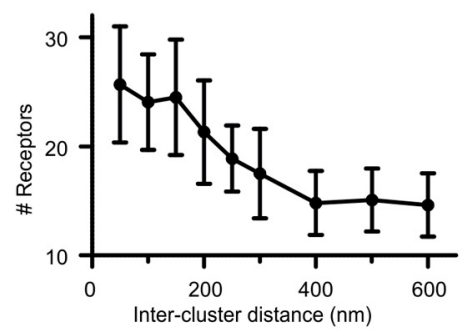

C

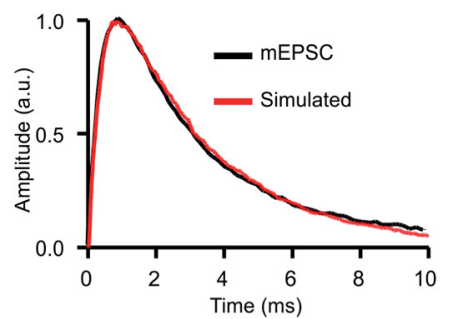

F

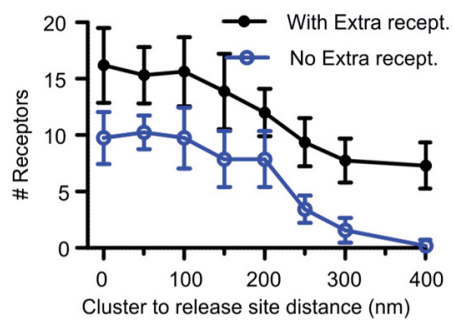

I

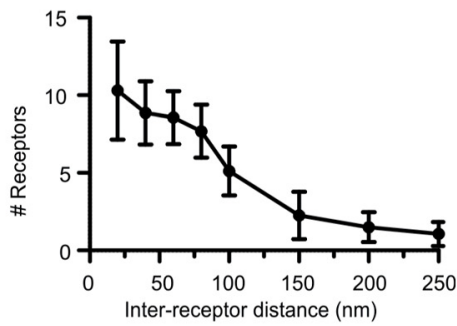

K2

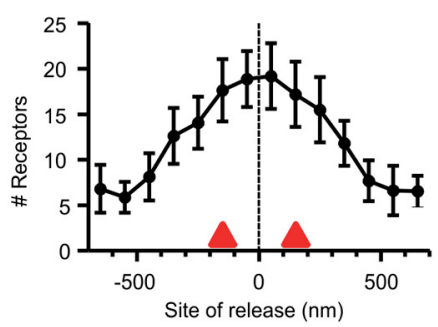

Figure 10. Modeling the role of AMPAR nanodomain in synaptic transmission. $A$, Kinetic AMPAR state diagram used for the Monte Carlo simulations performed essentially as described previously (Heine et al., 2008) and in Materials and Methods. AMPAR kinetics were modeled using Monte Carlo simulation (Glavinovic and Rabie, 1998; Franks et al., 2002; Raghavachari and Lisman, 2004; Heine et al., 2008) using a 7 state model and published values for kinetics rate constants, adjusted to fit the experimentally recorded mEPSCs in our system. In this scheme, resting AMPARs are in a C1 state and move to states C2 and C 3 upon successive binding of two glutamate molecules. 04 is the open state, whereas D5, D6, and D7 are desensitized states. Mono-liganded D7 desensitized AMPARs can move to the biliganded D6 state. B1, Schematic drawing of clustered AMPAR arrangement with respect to glutamate release. A cluster of 25 (5 $\times 5$ ) AMPAR (red dots) is positioned in front the glutamate release site, spaced by $20 \mathrm{~nm}$ in basal conditions. Extracluster receptors are disposed regularly spaced at $100 \mathrm{~nm}$ intervals (blue dots), and $3000 \mathrm{glutamate}$ (green halo) molecules are released on top of the cluster at time 0 in the presynaptic cell lying $15 \mathrm{~nm}$ away from the postsynapse. Glutamate was allowed to diffuse at $0.1 \mu \mathrm{m}^{2} \cdot \mathrm{ms}^{-1}$ as noted previously (Nielsen et al., 2004; Budisantoso et al., 2012) in the synaptic cleft (15 nm wide). B2, Color-coded plot of the cumulative probability of AMPAR opening within 10 ms after release of a vesicle containing 3000 glutamate molecules (arbitrary units) obtained by averaging 32 sweeps. The diameter of the activated area has a FWHM $\sim 160 \mathrm{~nm}$. C, Comparison of experimentally recorded spontaneous mEPSCs (average of 1700 individual events from 17 cells) and simulated EPSCs (average of 32 sweeps) obtained in conditions as in B1. Both curves are normalized for comparison. See below and Figure 9 for the respective amplitudes in various conditions of simulations or recording. $\boldsymbol{D}-\boldsymbol{F}$, Variation of $\mathrm{mEPSC}$ amplitude as the site of glutamate release is moved away from the nanocluster localization, as schematized in $\boldsymbol{D}$. Receptors are distributed as in $\boldsymbol{B} 1$. Sample mEPSCs (averages of 16 runs) are represented in $\boldsymbol{E}$, and the mean number of open AMPAR \pm SD in individual runs is presented in $\boldsymbol{F}$ in the presence (black circles) or absence (blue squares) of receptors outside the nanoclusters (i.e., with or without the blue dots in the $\mathbf{D}$ scheme). $\mathbf{G}-\mathbf{I}$, Dependence of mEPSCs on the AMPAR packing density, as schematized in $\boldsymbol{G}$. Series of simulated AMPAR-mediated mEPSCs (average of 16 trials) as a function of receptor packing interval (in nanometers) are represented in $\boldsymbol{H}$, and the mean number of open AMPAR \pm SD in individual runs is presented in $\mathbf{I . J 1}, \mathbf{J} \mathbf{2}$, Variation of $\mathrm{mEPSC}$ amplitude when two equivalent nanoclusters are present in the synapse at varying distance. Glutamate (3000 molecules) is released on top of the first cluster ( $5 \times 5$ receptors), whereas a second cluster of identical size is positioned at varying distances, as schematized in $\mathbf{J 1}$. The mean number of open AMPAR $\pm S D$ in individual runs as the function of the intercluster distance is presented in $\mathbf{J} \mathbf{2} \boldsymbol{K} \mathbf{1}, \boldsymbol{K} \mathbf{2}$, Variation of mEPSCs amplitude when two equivalent nanoclusters are present in the synapse at a fixed distance of $300 \mathrm{~nm}$ one form the other, whereas 3000 glutamate molecules are released at varying locations, as schematized in $\boldsymbol{K} \mathbf{1}$. The mean number of open AMPAR \pm SD in individual runs as a function of the position of the release site with respect to the two clusters is presented in $\mathbf{K} \mathbf{2}$. Two red triangles represent the location of the clusters.

cellular scaffolds, such as PSD95. Stabilization of AMPARs in nanodomains is reversible in the time span of seconds, and diffusion outside nanodomains is as rapid as in the extrasynaptic membrane. This observation reaffirms that AMPARs are in a dynamic equilibrium between sites of retention, consis- tent with the notion that postsynaptic slots control the heterogeneity in receptor immobilization locations within the synapse (Lisman and Raghavachari, 2006).

The size of nanodomains is surprisingly homogeneous, suggesting that it may be set by the physicochemical properties of a 
limited number of interacting building blocks. This subsynaptic organization is linked to the homeostasis of PSD95, as an increase or decrease in PSD95 expression levels modifies the nanodomain characteristics. A decrease in endogenous PSD95 expression levels broadens the surface of nanodomains and decreases AMPAR content and mEPSC amplitude. This is likely the result of an alteration in the nanodomain structure and AMPAR destabilization. This is in agreement with the recent EM tomography observation of RNA interference knockdown of PSD95 leading to patchy loss of PSD material. This loss correlates with the loss of putative AMPAR-type structures in the PSD (Chen et al., 2011). In contrast, overexpression of PSD95 increases AMPAR nanodomain size, but not its total content (i.e., lowering AMPAR density in the nanodomain). PSD95 overexpression does not significantly change the mEPSC amplitude, in agreement with previous work (Ehrlich and Malinow, 2004). However, it should be noted that PSD95 is most likely not the only core organizer of nanodomains, in particular as AMPAR and PSD95 do not perfectly colocalize, and as knocking down PSD95 (this study) or disrupting the PSD95-Stargazin interaction (Sainlos et al., 2011) does not fully disrupt nanodomain organization or synaptic transmission.

Together, we find it particularly interesting that mEPSC amplitude is well correlated with AMPAR nanodomain total content and not their size. Indeed, the amplitude of unitary synaptic responses is determined largely by the number of receptors binding neurotransmitters after presynaptic terminal release. This parameter is directly linked to the q value, which is the size of a quantum of glutamate inside one vesicle. Previously, this quantum was thought to be sufficient to saturate all synaptic AMPARs. More recently, biophysical models of glutamatergic synaptic transmission have outlined that, because of the steep glutamate gradient in the synaptic cleft after vesicle release, AMPARs can only be activated in an area of $\sim 150 \mathrm{~nm}$ (Lisman et al., 2007; Wu et al., 2007; Budisantoso et al., 2012) around the release site. As a consequence, organization of AMPARs inside the PSD, together with their proximity with presynaptic release sites, tune the strength and the variability of synaptic responses (Franks et al., 2002; Franks et al., 2003). Existing models of synaptic transmission rely mostly on a classical uniform distribution of AMPARs at the PSD. It means that, even if the q value is not sufficient to saturate all the receptors of the synapse, the AMPAR response will be directly correlated with the size of the quantum: the bigger the quantum, the more receptors are opened. The demonstration that AMPARs are highly concentrated in nanodomains and that these domains are long lasting opens the new possibility that single vesicle release may activate a nanodomain, and this could represent a postsynaptic quantum. Synaptic transmission could then be powerfully regulated by the composition and the position of the nanodomains relative to the presynaptic release site.

To investigate this concept further, we measured the distance between nanodomains inside single spines by STORM and sptPALM and performed modeling. In PSDs bearing multiple nanodomains, only approximately half of them are closer one to the other by $<250 \mathrm{~nm}$. With respect to the area over which AMPARs are thought to be activated by glutamate (diameter of $<200 \mathrm{~nm}$ ) (Lisman et al., 2007), FWHM computed from our simulations $156 \pm 15 \mathrm{~nm}$ (Fig. 10B2), this implies that a large fraction of AMPAR nanodomains are functionally isolated one from the other with respect to single vesicle release (Fig. 10J1). Using Monte Carlo simulations, we also found that the distance between glutamate release site and an AMPAR nanodomain strongly impacts the efficacy of transmission. The identity, location, and number of vesicle release sites at individual terminals are still a matter of debate. Nevertheless, it is tempting to postulate a correlation between release sites and AMPAR nanodomains, likely through trans-synaptic adhesion proteins, such as neurexin/neuroligins.

Finally, previous work demonstrated that fast AMPAR diffusion in and out release sites renews the pool of naive receptors that can be activated and removes desensitized ones, allowing the synapse to sustain high-frequency stimulation (Heine et al., 2008). We demonstrated that this effect of AMPAR diffusion is possible only if AMPAR exchange takes place on a small area (diameter of a couple of hundred nanometers) or if AMPARs are moving rapidly. The concentration of AMPARs in nanodomains, and their high mobility outside these, provides a framework for sufficiently fast exchange in AMPARs as well as for changes in receptor density in front of release sites by fast aggregation and dissociation of nanodomains aided by lateral diffusion.

\section{References}

Bats C, Groc L, Choquet D (2007) The interaction between Stargazin and PSD-95 regulates AMPA receptor surface trafficking. Neuron 53:719734. CrossRef Medline

Baude A, Nusser Z, Molnár E, McIlhinney RA, Somogyi P (1995) Highresolution immunogold localization of AMPA type glutamate receptor subunits at synaptic and non-synaptic sites in rat hippocampus. Neuroscience 69:1031-1055. CrossRef Medline

Bernard V, Somogyi P, Bolam JP (1997) Cellular, subcellular, and subsynaptic distribution of AMPA-type glutamate receptor subunits in the neostriatum of the rat. J Neurosci 17:819-833. Medline

Budisantoso T, Matsui K, Kamasawa N, Fukazawa Y, Shigemoto R (2012) Mechanisms underlying signal filtering at a multisynapse contact. J Neurosci 32:2357-2376. CrossRef Medline

Cheezum MK, Walker WF, Guilford WH (2001) Quantitative comparison of algorithms for tracking single fluorescent particles. Biophys J 81:2378 2388. CrossRef Medline

Chen X, Winters C, Azzam R, Li X, Galbraith JA, Leapman RD, Reese TS (2008) Organization of the core structure of the postsynaptic density. Proc Natl Acad Sci U S A 105:4453-4458. CrossRef Medline

Chen X, Nelson CD, Li X, Winters CA, Azzam R, Sousa AA, Leapman RD, Gainer H, Sheng M, Reese TS (2011) PSD-95 is required to sustain the molecular organization of the postsynaptic density. J Neurosci 31:63296338. CrossRef Medline

Choquet D (2010) Fast AMPAR trafficking for a high-frequency synaptic transmission. Eur J Neurosci 32:250-260. CrossRef Medline

Dani A, Huang B, Bergan J, Dulac C, Zhuang X (2010) Superresolution imaging of chemical synapses in the brain. Neuron 68:843-856. CrossRef Medline

Ehlers MD, Heine M, Groc L, Lee MC, Choquet D (2007) Diffusional trapping of GluR1 AMPA receptors by input-specific synaptic activity. Neuron 54:447-460. CrossRef Medline

Ehrlich I, Malinow R (2004) Postsynaptic density 95 controls AMPA receptor incorporation during long-term potentiation and experience-driven synaptic plasticity. J Neurosci 24:916-927. CrossRef Medline

El-Husseini AE, Schnell E, Chetkovich DM, Nicoll RA, Bredt DS (2000) PSD-95 involvement in maturation of excitatory synapses. Science 290: 1364-1368. Medline

Fölling J, Bossi M, Bock H, Medda R, Wurm CA, Hein B, Jakobs S, Eggeling C, Hell SW (2008) Fluorescence nanoscopy by ground-state depletion and single-molecule return. Nat Methods 5:943-945. CrossRef Medline

Franks KM, Bartol TM Jr, Sejnowski TJ (2002) A Monte Carlo model reveals independent signaling at central glutamatergic synapses. Biophys J 83: 2333-2348. CrossRef Medline

Franks KM, Stevens CF, Sejnowski TJ (2003) Independent sources of quantal variability at single glutamatergic synapses. J Neurosci 23:3186-3195. Medline

Frischknecht R, Heine M, Perrais D, Seidenbecher CI, Choquet D, Gundelfinger ED (2009) Brain extracellular matrix affects AMPA recep- 
tor lateral mobility and short-term synaptic plasticity. Nat Neurosci 12:897-904. CrossRef Medline

Fukazawa Y, Shigemoto R (2012) Intra-synapse-type and inter-synapsetype relationships between synaptic size and AMPAR expression. Curr Opin Neurobiol 22:446-452. CrossRef Medline

Ganeshina O, Berry RW, Petralia RS, Nicholson DA, Geinisman Y (2004) Differences in the expression of AMPA and NMDA receptors between axospinous perforated and nonperforated synapses are related to the configuration and size of postsynaptic densities. J Comp Neurol 468: 86-95. CrossRef Medline

Giannone G, Hosy E, Levet F, Constals A, Schulze K, Sobolevsky AI, Rosconi MP, Gouaux E, Tampé R, Choquet D, Cognet L (2010) Dynamic superresolution imaging of endogenous proteins on living cells at ultra-high density. Biophys J 99:1303-1310. CrossRef Medline

Glavinovíc MI (1999) Monte Carlo simulation of vesicular release, spatiotemporal distribution of glutamate in synaptic cleft and generation of postsynaptic currents. Pflugers Arch 437:462-470. CrossRef Medline

Glavinovíc MI, Rabie HR (1998) Monte Carlo simulation of spontaneous miniature excitatory postsynaptic currents in rat hippocampal synapse in the presence and absence of desensitization. Pflugers Arch 435:193-202. Medline

Groc L, Heine M, Cognet L, Brickley K, Stephenson FA, Lounis B, Choquet D (2004) Differential activity-dependent regulation of the lateral mobilities of AMPA and NMDA receptors. Nat Neurosci 7:695-696. CrossRef Medline

Heilemann M, Margeat E, Kasper R, Sauer M, Tinnefeld P (2005) Carbocyanine dyes as efficient reversible single-molecule optical switch. J Am Chem Soc 127:3801-3806. CrossRef Medline

Heilemann M, van de Linde S, Schüttpelz M, Kasper R, Seefeldt B, Mukherjee A, Tinnefeld P, Sauer M (2008) Subdiffraction-resolution fluorescence imaging with conventional fluorescent probes. Angew Chem Int Ed Engl 47:6172-6176. CrossRef Medline

Heine M, Groc L, Frischknecht R, Béique JC, Lounis B, Rumbaugh G, Huganir RL, Cognet L, Choquet D (2008) Surface mobility of postsynaptic AMPARs tunes synaptic transmission. Science 320:201-205. CrossRef Medline

Hell SW (2009) Microscopy and its focal switch. Nat Methods 6:24-32. CrossRef Medline

Hoze N, Nair D, Hosy E, Sieben C, Manley S, Herrmann A, Sibarita JB, Choquet D, Holcman D (2012) Heterogeneity of AMPA receptor trafficking and molecular interactions revealed by superresolution analysis of live cell imaging. Proc Natl Acad Sci U S A 109:17052-17057. CrossRef Medline

Izeddin I, Boulanger J, Racine V, Specht CG, Kechkar A, Nair D, Triller A, Choquet D, Dahan M, Sibarita JB (2012) Wavelet analysis for single molecule localization microscopy. Opt Express 20:2081-2095. CrossRef Medline

Jonas P, Major G, Sakmann B (1993) Quantal components of unitary EPSCs at the mossy fiber synapse on CA3 pyramidal cells of the rat hippocampus. J Physiol 472:615-663. Medline

Kaech S, Banker G (2006) Culturing hippocampal neurons. Nat Protoc 1:2406-2415. CrossRef Medline

Kechkar A, Nair D, Heilemann M, Choquet D, Sibarita JB (2013) Real-time analysis and visualization for single-molecule based super-resolution microscopy. PloS One 8:e62918. CrossRef Medline

Kharazia VN, Weinberg RJ (1997) Tangential synaptic distribution of NMDA and AMPA receptors in rat neocortex. Neurosci Lett 238:41-44. CrossRef Medline

Kharazia VN, Phend KD, Rustioni A, Weinberg RJ (1996) EM colocalization of AMPA and NMDA receptor subunits at synapses in rat cerebral cortex. Neurosci Lett 210:37-40. CrossRef Medline

Kubitscheck U, Homann U, Thiel G (2000) Osmotically evoked shrinking of guard-cell protoplasts causes vesicular retrieval of plasma membrane into the cytoplasm. Planta 210:423-431. CrossRef Medline

Lisman J, Raghavachari S (2006) A unified model of the presynaptic and postsynaptic changes during LTP at CA1 synapses. Sci STKE 2006:re11. CrossRef Medline

Lisman JE, Raghavachari S, Tsien RW (2007) The sequence of events that underlie quantal transmission at central glutamatergic synapses. Nat Rev Neurosci 8:597-609. CrossRef Medline

Liu G, Choi S, Tsien RW (1999) Variability of neurotransmitter concentra- tion and nonsaturation of postsynaptic AMPA receptors at synapses in hippocampal cultures and slices. Neuron 22:395-409. CrossRef Medline Lüscher C, Xia H, Beattie EC, Carroll RC, von Zastrow M, Malenka RC, Nicoll RA (1999) Role of AMPA receptor cycling in synaptic transmission and plasticity. Neuron 24:649-658. CrossRef Medline

Macgillavry HD, Song Y, Raghavachari S, Blanpied TA (2013) Nanoscale scaffolding domains within the postsynaptic density concentrate synaptic AMPA receptors. Neuron 78:615-622. CrossRef Medline

Makino H, Malinow R (2009) AMPA receptor incorporation into synapses during LTP: the role of lateral movement and exocytosis. Neuron 64:381390. CrossRef Medline

Manley S, Gillette JM, Patterson GH, Shroff H, Hess HF, Betzig E, Lippincott-Schwartz J (2008) High-density mapping of singlemolecule trajectories with photoactivated localization microscopy. Nat Methods 5:155-157. CrossRef Medline

Masugi-Tokita M, Shigemoto R (2007) High-resolution quantitative visualization of glutamate and GABA receptors at central synapses. Curr Opin Neurobiol 17:387-393. CrossRef Medline

Masugi-Tokita M, Tarusawa E, Watanabe M, Molnár E, Fujimoto K, Shigemoto R (2007) Number and density of AMPA receptors in individual synapses in the rat cerebellum as revealed by SDS-digested freeze-fracture replica labeling. J Neurosci 27:2135-2144. CrossRef Medline

Matsubara A, Laake JH, Davanger S, Usami S, Ottersen OP (1996) Organization of AMPA receptor subunits at a glutamate synapse: a quantitative immunogold analysis of hair cell synapses in the rat organ of Corti. J Neurosci 16:4457-4467. Medline

Mondin M, Labrousse V, Hosy E, Heine M, Tessier B, Levet F, Poujol C, Blanchet C, Choquet D, Thoumine O (2011) Neurexin-neuroligin adhesions capture surface-diffusing AMPA receptors through PSD-95 scaffolds. J Neurosci 31:13500-13515. CrossRef Medline

Nicoll RA, Tomita S, Bredt DS (2006) Auxiliary subunits assist AMPA-type glutamate receptors. Science 311:1253-1256. CrossRef Medline

Nielsen TA, DiGregorio DA, Silver RA (2004) Modulation of glutamate mobility reveals the mechanism underlying slow-rising AMPAR EPSCs and the diffusion coefficient in the synaptic cleft. Neuron 42 : 757-771. CrossRef Medline

Nusser Z, Mulvihill E, Streit P, Somogyi P (1994) Subsynaptic segregation of metabotropic and ionotropic glutamate receptors as revealed by immunogold localization. Neuroscience 61:421-427. CrossRef Medline

Nusser Z, Lujan R, Laube G, Roberts JD, Molnar E, Somogyi P (1998) Cell type and pathway dependence of synaptic AMPA receptor number and variability in the hippocampus. Neuron 21:545-559. CrossRef Medline

Opazo P, Labrecque S, Tigaret CM, Frouin A, Wiseman PW, De Koninck P, Choquet D (2010) CaMKII triggers the diffusional trapping of surface AMPARs through phosphorylation of Stargazin. Neuron 67: 239-252. CrossRef Medline

Opazo P, Sainlos M, Choquet D (2012) Regulation of AMPA receptor surface diffusion by PSD-95 slots. Curr Opin Neurobiol 22:453-460. CrossRef Medline

Penn AC, Balik A, Wozny C, Cais O, Greger IH (2012) Activity-mediated AMPA receptor remodeling, driven by alternative splicing in the ligandbinding domain. Neuron 76:503-510. CrossRef Medline

Petersen JD, Chen X, Vinade L, Dosemeci A, Lisman JE, Reese TS (2003) Distribution of postsynaptic density (PSD)- 95 and $\mathrm{Ca}^{2+} / \mathrm{calmodulin}-$ dependent protein kinase II at the PSD. J Neurosci 23:11270-11278. Medline

Petralia RS, Wang YX, Mayat E, Wenthold RJ (1997) Glutamate receptor subunit 2-selective antibody shows a differential distribution of calciumimpermeable AMPA receptors among populations of neurons. J Comp Neurol 385:456-476. CrossRef Medline

Petrini EM, Lu J, Cognet L, Lounis B, Ehlers MD, Choquet D (2009) Endocytic trafficking and recycling maintain a pool of mobile surface AMPA receptors required for synaptic potentiation. Neuron 63:92-105. CrossRef Medline

Racine V, Hertzog A, Jouanneau J, Salamero J, Kervrann C, Sibarita JB (2006) Multiple-target tracking of 3D fluorescent objects based on simulated annealing. IEEE International Symposium on Biomedical Imaging: Macro to nano. Vol 1-3:1020-1023. CrossRef

Racine V, Sachse M, Salamero J, Fraisier V, Trubuil A, Sibarita JB (2007) Visualization and quantification of vesicle trafficking on a threedimensional cytoskeleton network in living cells. J Microsc 225:214-228. CrossRef Medline 
Raghavachari S, Lisman JE (2004) Properties of quantal transmission at CA1 synapses. J Neurophysiol 92:2456-2467. CrossRef Medline

Saglietti L, Dequidt C, Kamieniarz K, Rousset MC, Valnegri P, Thoumine O, Beretta F, Fagni L, Choquet D, Sala C, Sheng M, Passafaro M (2007) Extracellular interactions between GluR2 and N-cadherin in spine regulation. Neuron 54:461-477. CrossRef Medline

Sainlos M, Tigaret C, Poujol C, Olivier NB, Bard L, Breillat C, Thiolon K, Choquet D, Imperiali B (2011) Biomimetic divalent ligands for the acute disruption of synaptic AMPAR stabilization. Nat Chem Biol 7: 81-91. CrossRef Medline

Schlüter OM, Xu W, Malenka RC (2006) Alternative N-terminal domains of PSD-95 and SAP97 govern activity-dependent regulation of synaptic AMPA receptor function. Neuron 51:99-111. CrossRef Medline

Schnell E, Sizemore M, Karimzadegan S, Chen L, Bredt DS, Nicoll RA (2002) Direct interactions between PSD-95 and stargazin control synaptic AMPA receptor number. Proc Natl Acad Sci U S A 99:13902-13907. CrossRef Medline

Sharma K, Fong DK, Craig AM (2006) Postsynaptic protein mobility in dendritic spines: long-term regulation by synaptic NMDA receptor activation. Mol Cell Neurosci 31:702-712. CrossRef Medline

Takumi Y, Matsubara A, Rinvik E, Ottersen OP (1999a) The arrangement of glutamate receptors in excitatory synapses. Ann N Y Acad Sci 868:474-482. CrossRef Medline
Takumi Y, Ramírez-León V, Laake P, Rinvik E, Ottersen OP (1999b) Different modes of expression of AMPA and NMDA receptors in hippocampal synapses. Nat Neurosci 2:618-624. CrossRef Medline

Tao-Cheng JH, Crocker VT, Winters CA, Azzam R, Chludzinski J, Reese TS (2011) Trafficking of AMPA receptors at plasma membranes of hippocampal neurons. J Neurosci 31:4834-4843. CrossRef Medline

Tarusawa E, Matsui K, Budisantoso T, Molnár E, Watanabe M, Matsui M, Fukazawa Y, Shigemoto R (2009) Input-specific intrasynaptic arrangements of ionotropic glutamate receptors and their impact on postsynaptic responses. J Neurosci 29:12896-12908. CrossRef Medline

van de Linde S, Löschberger A, Klein T, Heidbreder M, Wolter S, Heilemann M, Sauer M (2011) Direct stochastic optical reconstruction microscopy with standard fluorescent probes. Nat Protoc 6:9911009. CrossRef Medline

Wenthold RJ, Petralia RS, Blahos J II, Niedzielski AS (1996) Evidence for multiple AMPA receptor complexes in hippocampal CA1/CA2 neurons. J Neurosci 16:1982-1989. Medline

Wu XS, Xue L, Mohan R, Paradiso K, Gillis KD, Wu LG (2007) The origin of quantal size variation: vesicular glutamate concentration plays a significant role. J Neurosci 27:3046-3056. CrossRef Medline

Xu-Friedman MA, Regehr WG (2004) Structural contributions to shortterm synaptic plasticity. Physiol Rev 84:69-85. CrossRef Medline 